\title{
The diagnostic conundrum in non-alcoholic fatty liver disease
}

\author{
Valerio Rosato®, Mario Masarone* ${ }^{*}$, Andrea Aglitti®, Marcello Persico® \\ Internal Medicine and Hepatology Unit, Department of Medicine, Surgery and Odontostomatology "Scuola Medica Salernitana"- \\ University of Salerno, Street Salvador Allende, 43, Fisciano, 84084 Campania, Italy
}

*Correspondence: Mario Masarone, Internal Medicine and Hepatology Unit, Department of Medicine, Surgery and Odontostomatology “Scuola Medica Salernitana”-University of Salerno, Street Salvador Allende, 43, Fisciano, 84084 Campania, Italy.mmasarone@unisa.it

Academic Editor: Amedeo Lonardo, Azienda Ospedaliero-Universitaria di Modena, Italy

Received: June 7, 2020 Accepted: July 12, 2020 Published: October 30, 2020

Cite this article: Rosato V, Masarone V, Aglitti A, Persico M. The diagnostic conundrum in non-alcoholic fatty liver disease. Explor Med. 2020;1:259-86. https://doi.org/10.37349/emed.2020.00018

\begin{abstract}
Non-alcoholic fatty liver disease (NAFLD) has become the most common liver alteration worldwide. It encompasses a spectrum of disorders that range from simple steatosis to a progressive form, defined non-alcoholic steatohepatitis (NASH), that can lead to advanced fibrosis and eventually cirrhosis and hepatocellular carcinoma. On liver histology, NASH is characterized by the concomitant presence of significant fat accumulation and inflammatory reaction with hepatocellular injury. Until now, liver biopsy is still required to differentiate simple steatosis from NASH and evaluate the degree of liver fibrosis. Unfortunately, this technique has well-known limitations, including invasiveness and expensiveness. Moreover, it may be biased by sampling error and intra- or inter-observed variability. Furthermore, due to the increasing prevalence of NAFLD worldwide, to program a systematic screening with liver biopsy is not imaginable. In recent years, different techniques were developed and validated with the aim of non-invasively identifying NASH and assess liver fibrosis degrees. The non-invasive tests range from simple blood-tests analyses to composite scores and complex imaging techniques. Nevertheless, even if they could represent cost-effective strategies for diagnosing NASH, advanced fibrosis and cirrhosis, their accuracy and consequent usefulness are to be discussed. With this aim, in this review the authors summarize the current state of non-invasive assessment of NAFLD. In particular, in addition to the well-established tests, the authors describe the future perspectives in this field, reporting the latest tests based on OMICS, gut-miocrobioma and micro-RNAs. Finally, the authors provide an accurate assessment of how these non-invasive tools perform in clinical practice depending on the clinical context, with the aim of giving the clinicians a useful tool to try to resolve the diagnostic conundrum of NAFLD.
\end{abstract}

\section{Keywords}

Cirrhosis, non-alcoholic fatty liver disease, steatosis, non-alcoholic steatohepatitis, liver fibrosis, non invasive, serum biomarkers 


\section{Introduction}

In the last two decades, non-alcoholic fatty liver disease (NAFLD) has become the most common-liver disorder worldwide, because of the burden reduction of viral hepatitis together with the increase of metabolic derangements related to the "western lifestyle" [1]. NAFLD encompasses a spectrum of liver disorders of which the starting point is the "simple steatosis" [or non-alcoholic fatty liver (NAFL)], defined as the presence of a significant fat accumulation in the liver (> 5\% of hepatocytes), developed in absence of secondary causes (i.e. an "unsafe" quantity of alcohol consumption, medications or heritable conditions) [2]. The concomitant presence of an inflammatory reaction with hepatocellular injury defines the condition of non-alcoholic steatohepatitis (NASH), which is an evolutionary disease that could lead to advanced fibrosis (AF) and eventually cirrhosis and hepatocellular carcinoma (HCC) [3]. Alarmingly, the possibility of an HCC development in NASH patients has been reported also in the absence of cirrhosis [4].

It has been estimated that NAFLD has a prevalence of about $25 \%$ in the world adult population, reaching $75 \%$ in obese individuals and even more in patients with type 2 diabetes mellitus (T2DM). This estimate has been reported by several studies, based mostly on imaging techniques [ultrasound (US), computed tomography, and magnetic resonance imaging (MRI)/spectroscopy] [5, 6].

Evaluating the prevalence of NASH on general population is problematic, because its ideal diagnosis should require a liver biopsy which is, obviously, not routinely performed. However, in studies on biopsyproven NAFLD patients, in about $20 \%$ of the cases a NASH histology has been demonstrated [7]. Furthermore, in case a series of liver biopsies performed on healthy subjects (living donors for liver transplantations or volunteers), NASH was found in about $1.5-15 \%$ of subjects $[8,9]$. Therefore, on the basis of these evidences, a NASH prevalence on general population of 3-6\% could be indirectly estimated [2]. Nevertheless, it has to be pointed out that, if we analyze only specialized tertiary centers treating liver diseases, the estimates of the prevalence of NASH in NAFLD patients may be as high as 90.4\% [10].

Even if the most common cause of death among NAFLD patients is represented by cardiovascular disease (CVD), independently of presence of other metabolic comorbidities, NAFLD itself is becoming a major cause of liver-related mortality [11]. Patients with both simple steatosis or NASH may develop progressive liver fibrosis, but only NASH patients show a higher risk of rapid progression in AF [12]. Because of the close association with T2DM and obesity, it has been estimated that the prevalence of NASH will increase, causing a significant clinical and economic impact and poorer patient-reported outcomes [13]. In fact, at the moment, NASH is the first indication for liver transplantation in women and, probably, it will become soon the leading indication also in men, overtaking alcoholic liver disease [14]. Furthermore, in NASH liver transplant patients, it has been shown an increasing trend of the prevalence of HCC, higher than that for any other etiology [15].

Although international guidelines on this issue encourage an early identification of NAFLD patients, this disease has a completely asymptomatic course, especially in the initial stages. In this way, often the diagnosis happens incidentally and, unfortunately, often in an advanced stage. The routine radiological examinations, such as abdomen US, could easily detect this liver disorder but cannot satisfactorily discriminate NASH or liver fibrosis degrees [16].

The gold standard for NAFL and NASH discrimination still remains liver biopsy, but this technique is expensive and invasive, and it may be biased by sampling error and intra-observer and inter-observer variability [17]. Furthermore, the utility of biopsy is still controversial as no NAFLD-specific treatments have yet been approved, and the only generally recommended interventions for this condition are lifestyle modifications, regardless of the presence of a NASH or a simple steatosis [18]. In a recent multi-country preference study, conducted among 121 physicians managing NASH patients, it is reported that in about $57 \%$ of cases they are reluctant to perform confirmatory liver biopsies due to these limited therapeutic options together with patient refusal, despite the fact that $81 \%$ of them reported performing liver biopsy [19].

In recent years, increasing attention has been given to "NAFLD biomarkers" and several non-invasive diagnostic methods have been developed, ranging from simple blood-based tests to composite scores and complex imaging techniques, with the aim of precisely, and non-invasively, identifying NAFLD and assessing liver fibrosis degrees [20]. The application of non-invasive tests for NAFLD is aimed at the discrimination of 
simple steatosis from steatohepatitis, given their different prognosis, and the evaluation of fibrosis, in order to guide the management decisions $[1,21]$.

The objective of this review is to evaluate the available data on this topic and analyze the reliability of non-invasive tests for NAFLD, in order to offer a guide to the clinicians evaluating NAFLD patients to help them in untangling the NAFLD diagnostic conundrum.

\section{The clinical problem}

\section{Who should we screen?}

Identifying NAFLD or NASH may be a complex challenge to face, as these diseases almost always have an asymptomatic course [22]. The symptoms usually reported are completely non-specific, as fatigue or vague abdominal pain and, if cirrhosis has not yet been developed, a physical examination is typically unrevealing. Very often liver US is the imagining exam that incidentally identifies a hepatic steatosis, most of the time in patients with normal liver function tests, but also, occasionally, in subjects that are already in an advanced stage [23].

NAFLD is strongly associated with features of metabolic syndrome (MetS) (obesity, dyslipidemia, T2DM, and hypertension), and vice-versa these conditions increase the risk of developing NAFLD [24]. In fact, NAFLD is often referred to as the "hepatic manifestation" of MetS, even though it has been demonstrated that NAFLD may precede the development of an overt MetS or T2DM [6]. In a meta-analysis on 20 prospective studies (117, 020 patients followed for a median time of 5 years), it has been shown that the presence of NAFLD, diagnosed by either liver enzymes or ultrasonography, is associated with a relative risk of incident T2DM or MetS ranging between 1.58-1.97 and 1.8-3.2 respectively [25]. Similarly, in a more recent metaanalysis on 19 observational studies (296, 439 individuals), a higher risk of incident diabetes (hazard ratio 2.2) has been shown in patients with NAFLD compared to those without NAFLD [26]. However, the pathophysiologic relationship between NAFLD and insulin resistance is still not completely clear, as demonstrated by the fact that there are epidemiological studies showing that NAFLD is not invariably associated with MetS [27]. Moreover, several evidences showed an association between NAFLD and other extra-hepatic manifestations (endocrinopathies, osteoporosis, polycystic ovarian syndrome, psoriasis, and sleep apnea) [11]. Furthermore, NAFLD has been independently associated with fatal/non-fatal CVD and arrhythmic complications, including atrial fibrillation [28].

As an evidence of the close correlation between NAFLD and MetS or T2DM, it has been shown that the coexistence of these two last conditions increases the risk of NASH and, consequently, worsens liver fibrosis [16]. As a proof, in a study on 118 biopsy-proven NAFLD patients, insulin resistance has been associated with the whole pathological spectrum of NAFLD, notably including fibrosis which dictates the long-term prognosis of NAFLD [29, 30].

It has been described that $5-20 \%$ of patients with NAFLD are neither overweight nor obese, defining the so-called metabolically obese normal-weight individuals, who are at high risk of liver damage and cardiovascular events despite the normal weight [31]. In a recent retrospective study on 1, 000 biopsyproven NAFLD patients, it has been reported that the subjects with a body mass index (BMI) $>25 \mathrm{~kg} / \mathrm{m}^{2}$ have no differences in histological disease severity compared to patients with a BMI $<25 \mathrm{~kg} / \mathrm{m}^{2}$ [32].

Therefore, it is difficult to define which patient deserves an in-depth screening for NAFLD. A recent costeffectiveness analysis on the utility of screening for NASH among diabetic patients showed an improvement in liver-related outcomes, but a lack of cost-effectiveness due to the side effects of the selected treatment (pioglitazone) [33]. However, the study suggested that the screening for NASH may be cost-effective as soon as it will be available specific medications with milder side effects [33]. Thus, the recommendations of the international guidelines do not advise a routine screening for NAFLD in high-risk populations (diabetes, obesity et cetera), but only in patients with symptoms or signs attributable to liver disease, abnormal liver biochemistry or incidental detection of hepatic steatosis on imaging. These last subjects should be evaluated as "suspect NAFLD" and assessed for liver fibrosis, metabolic risk factors or alternate causes for fatty liver (alcohol, medications et cetera) $[2,18]$. Moreover, if a NAFLD diagnosis is made, a systematic screening of 
family members of the diagnosed subjects is not currently recommended, despite several evidences from studies on twin's cohorts which suggest a familial clustering of NAFLD [34].

A new definition for NAFLD has been recently suggested, to better highlight the close relationship with MetS, namely the "metabolic associated fatty liver disease (MAFLD)", proposing also new "positive" criteria for diagnosis (overweight/obesity, T2DM or evidences of metabolic dysregulation), regardless of alcohol consumption or other concomitant liver disease [35] (Figure 1). These new diagnostic criteria would allow recognizing straightforwardly the metabolic liver changes coexisting with other conditions, but also clarifying the most appropriate method of identifying NAFLD patients [35]. However, further clear recommendations of the international guidelines are necessary in order to establish the appropriate screening strategies, following this "update".
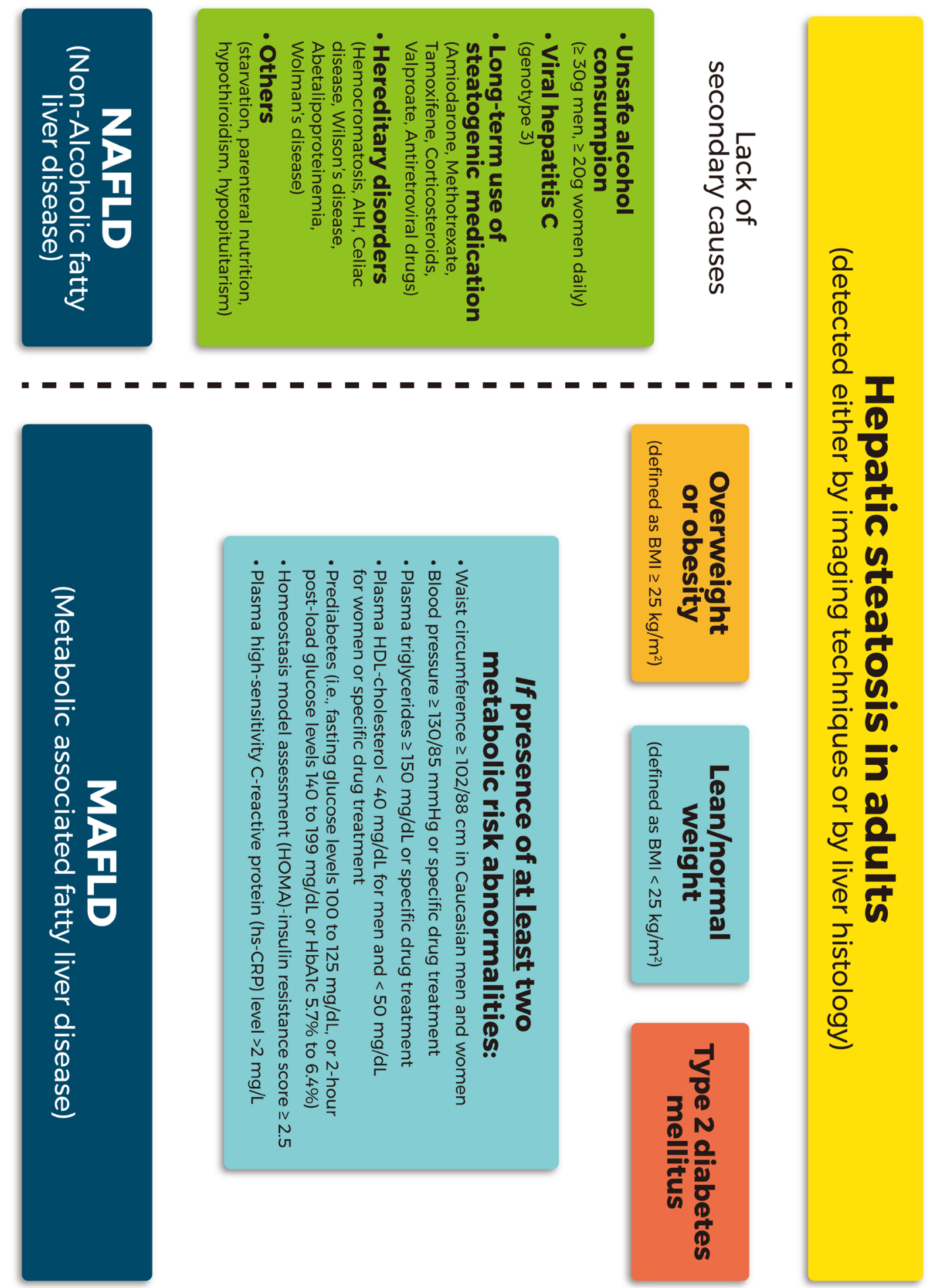

Figure 1. Comparison between the flowchart for the diagnostic criteria for NAFLD and the new proposed "positive" diagnostic criteria for MAFLD. AlH: autoimmune hepatitis; HDL: high density lipoprotein 
A proposed diagnostic and subsequent follow-up flowchart, which is based on the European Association for the Study of the Liver (EASL), American Association for the Study of Liver Diseases (AASLD) guidelines and clinical experiences, is presented in Figure 2.

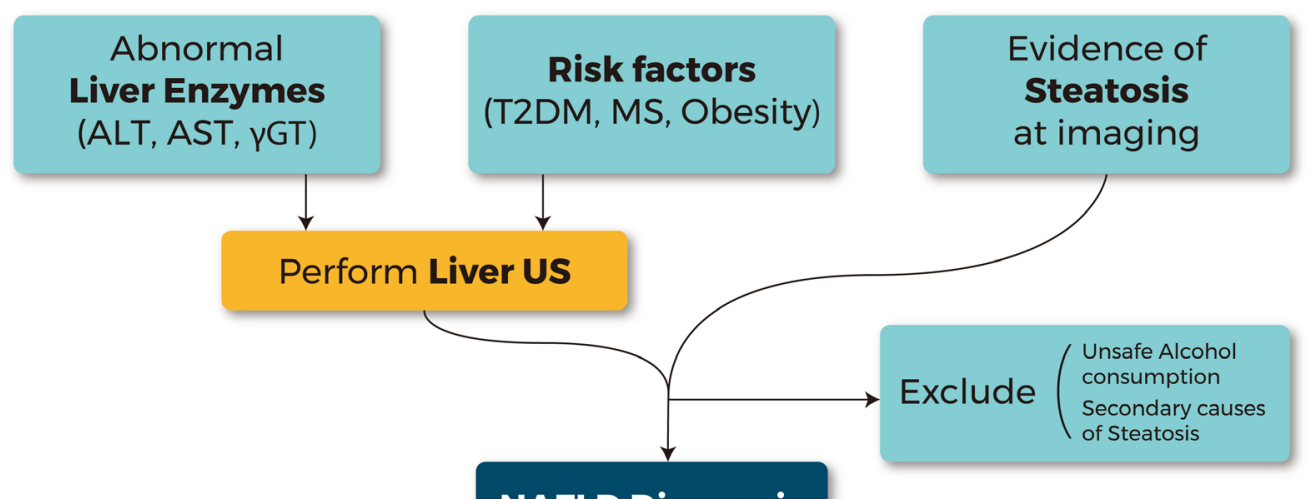

NAFLD Diagnosis

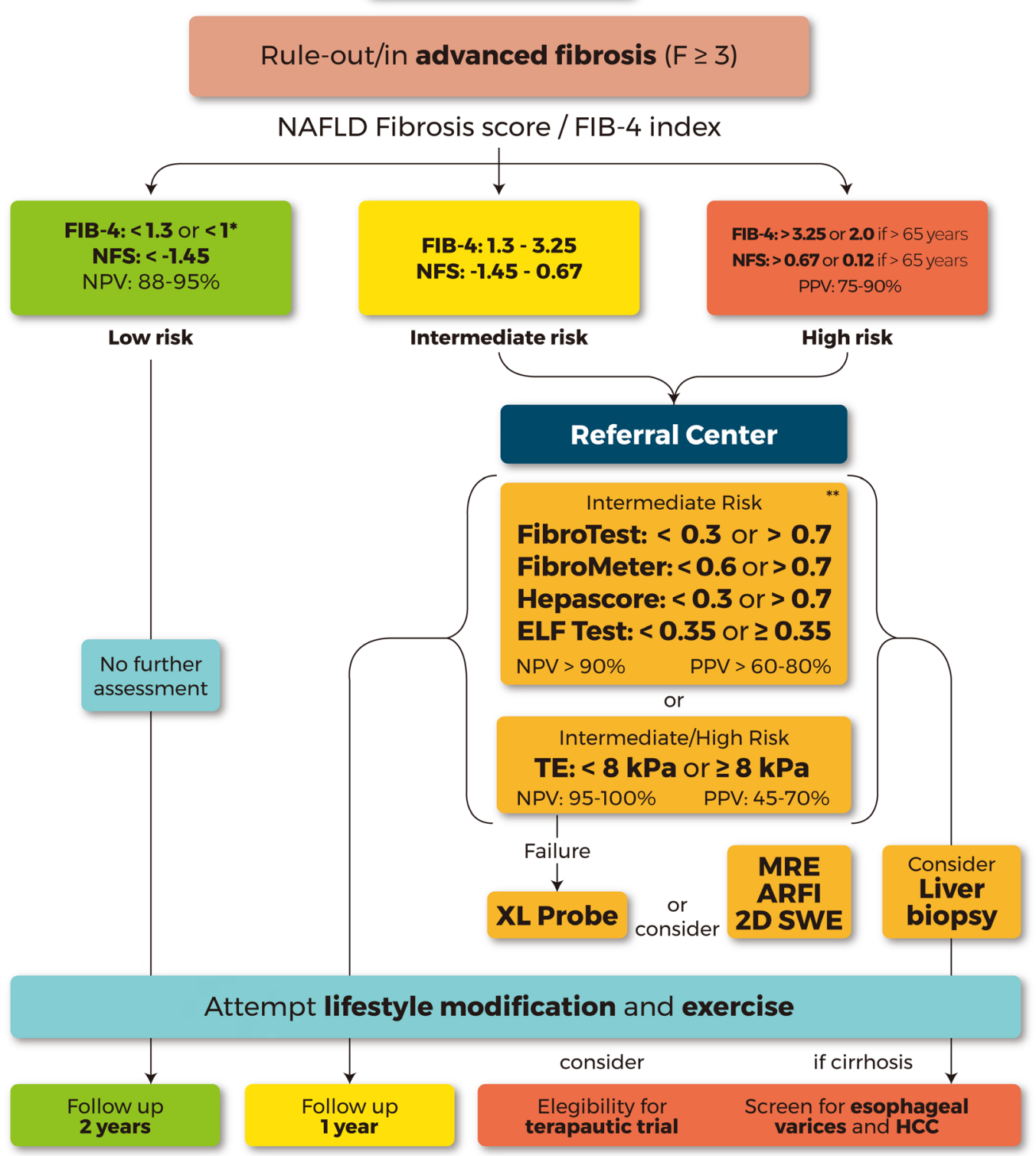

Figure 2. A proposed algorithm to be used by clinicians to perform the diagnosis and the risk stratification of patients with steatosis by means of non-invasive tests. The suspicion of NAFLD should be made in patients with metabolic risk factors [T2DM, obesity or MetS or abnormal liver test (AST, ALT, and GGT)]. The evidence of steatosis, identified at US imaging, allows NAFLD diagnosis only after excluding any secondary cause of steatosis. The subsequent risk stratification and the choice of the non-invasive test should be guided by the clinical context and, therefore, by local availability. In primary health-care settings, NFS and FIB-4, simple and inexpensive, should be used as first line, allowing ruling out patient with intermediate/high risk of AF with a high NPV (88-95\%). FIB-4 $<1.3$ or NFS $<-1.45$ identify low risk patients with simple NAFL that don't need further assessments and may eventually 
undergo to follow-up at 2-3 years. In order to exclude also F2 patients, in general populations settings, a FIB-4 cutoff <1, able to rule out any fibrosis (F0 vs. F1-4), was recently proposed. The identified patients with intermediate (FIB-4 1.3-3.25, NFS 1.450.67 ) or high risk (FIB-4 $>3.2$ or NFS $>0.67$ ) of AF should be referred to a tertiary center for a "second-line evaluation" with more specific non-invasive tests. In patients with intermediate risk, patented serum biomarkers [FibroTest (FT), FibroMeter, Hepascore, ELF test] could be considered to discriminate low-risk patients with good diagnostic accuracy (NPV > 90\%), when (and if) it should be possible to obtain the funds to bear the costs. TE is the most widely available and best validated technique. At values less than $8 \mathrm{kPa}$, measured by M-probe, it allows excluding intermediate or high-risk patients with high accuracy (NPV 95-100\%). $\mathrm{XL}$-probe may reduce the TE failure rate, especially in patients with a skin-liver capsule distance $>25 \mathrm{~mm}$. Other techniques as 2D-SWE and ARFI or MRE may be considered according to local availability (particularly in obese patients, with $\mathrm{BMI}>35 \mathrm{~kg} / \mathrm{m}^{2}$ ). In patients with intermediate $(8-10 \mathrm{kPa}$ ) or high risk (> $10 \mathrm{kPa}$, PPV 47-70\%) for AF a further assessment by liver biopsy should be considered. Lifestyle modification and exercise should be suggested to all patients with NAFLD. Patients with AF or cirrhosis should be also screened for esophageal varices and HCC. In addition, the eligibility for therapeutic trials should be taken into consideration. * addressed to rule out any fibrosis (F0 vs. F1-4); ${ }^{* *}$ patented test; ALT: alanine transaminase; AST: aspartate transaminase; FIB-4: fibrosis 4 index; ELF: enhanced liver fibrosis; GGT: gamma-glutamil transpeptidase; NFS: NAFLD fibrosis score; NPV: negative predictive value; TE: transient elastography; PPV: positive predictive value; 2D-SWE: two-dimensional shear wave elastography; ARFI: acoustic radiation force impulse; MRE: magnetic resonance elastography

\section{How do and what we assess?}

Once a NAFLD has been identified and other coexisting conditions of chronic liver disease (CLD) excluded, an assessment of the presence of NASH and the degree of liver fibrosis is required. As previously mentioned, the gold-standard method to make a diagnosis of NASH is by performing liver biopsy. Nevertheless, several noninvasive tests, based on imaging and biochemistry, have been proposed and evaluated to differentiate NASH from simple steatosis and to graduate liver fibrosis.

\section{Biomarkers for NASH diagnosis}

Several biomarkers, ranging from clinical parameters (gender, age, BMI, diabetes), liver enzymes (transaminases, bilirubin, ferritin), metabolic [insulin, homeostatic model assessment of insulin resistance, (HOMA-IR)], or lipid [cholesterol, triglycerides (TG)] markers, have been investigated in their accuracy to predict NASH. Predictably, given the complex pathogenesis of this disorder (including inflammation, oxidative stress, apoptosis, lipid and glucose metabolism), a single biomarker is not able, per se, to satisfactorily discriminate between NAFLD and NASH, therefore more complex models and scores based on multiple variables have been developed.

Several studies have shown that high values of ALT and AST are associated with higher risk of NASH, but, unfortunately, also normal values of transaminases were found in patients with NASH and/or advanced liver fibrosis [36]. In an Italian study on 458 NAFLD biopsy-proven patients, NASH was diagnosed in $59 \%$ of patients with normal ALT [37]. Moreover, it has been shown that NAFLD patients with normal aminotransferase levels are characterized by a prevalence of AF similar to that found in patients with elevated aminotransferase [38]. In fact, the conventional ALT cutoff level has shown only $72 \%$ sensitivity (Se) and $51 \%$ specificity (Sp) for the diagnosis of NASH [39]. Also, GGT and alkaline phosphatase were proposed to diagnose NASH, but their use alone has not shown an acceptable diagnostic reliability [40-42].

Therefore, in order to differentiate NASH from NAFL by means of routine serum biomarkers, different predictive models have been developed, in which multiple biomarkers were combined to improve the diagnostic accuracy $[43,44]$. The HAIR score has been proposed, it is determined using 3 parameters (hypertension, ALT, insulin resistance), and in its presenting study, showed an Se $80 \%$ and an Sp of 89\%, with an area under the receiver operating curve (AUROC) of 0.9, for NASH [45]. Despite these first promising results, subsequently, it revealed an underperformance in other populations, for example in diabetic patients, reaching unsatisfactory AUROCs [46]. The FT and Acti-Test (AT), composed of fibrosis indexes (alfa2macroglobulin, apolipoprotein A1, haptoglobin, total bilirubin and GGT) and necro-inflammatory indexes (FT plus ALT), were initially patented for the assessment of liver fibrosis, showing an AUROC of 0.88 [47]. Subsequently, in order to identify NASH, the "NashTest" (combing FT-AT plus weight, height, AST, glucose, TG, cholesterol adjusted for age and gender) has been developed with an estimated AUROC of 0.79 [48]. While NashTest and FT were also validated in population of patients with severe obesity and hyperlipidemia, maintaining a reasonable reliability for diagnosis of NASH $[49,50]$, in patients with type 2 diabetes FT-AT and NashTest underperformed, showing a lower AUROC of about 0.7 [51]. 
Interleukin-6 (IL-6) has been investigated as a putative marker of NASH. IL-6 is an inflammatory cytokine that may increase in NASH (and in other inflammatory conditions) but it is also involved in anti-inflammatory activity and in metabolic or regenerative processes [52]. The use of IL-6 to identify NASH was evaluated alone and within a predictive model together with other biomarkers, showing a good AUROC ranging from 0.79 to $0.9[53,54]$.

Elevated circulating levels of adiponectin were associated with elevated values of other inflammatory cytokines (IL-6, TNF-a) and with the presence of metabolic diseases (obesity, insulin resistance, dyslipidemia) [42]. Thus, adiponectin was evaluated in order to differentiate NASH and NAFL in a panel including also leptin and ghrelin, showing an AUROC of 0.79 [55].

Cytokeratin 18 (CK18) is a marker of cell death, the major hepatic intermediate filament protein cleaved by caspases during hepatocyte's apoptosis, and his usefulness for NASH diagnosis was studied for the cleaved (CK18-M30) or intact form (CK18-M65). CK18-M30 has been extensively studied and, in two meta-analyses, it was reported as an Se of $66-78 \%$ and Sp of $82-87 \%$, with a pooled AUROC of 0.82 for discriminating NASH [56-58]. The use of the CK18 was also evaluated within combinatorial models including clinical parameters (diabetes, gender, BMI) [59, 60], routine blood markers [ALT, platelets (PLT), TG] [61], adipocytokines and the combination of two isoforms of CK-18 [62]. Recently, in a study on 345 biopsy-proven NAFLD patients, the use in combination CK18-M30 and Golgi protein 73 (G-NASH model) has shown good accuracy for predicting NASH in patients with persistent normal ALT, with an AUROC of 0.84 [63]. Despite the fact that CK-18 has been widely validated, its testing is still not commercially available.

Mac-2 binding protein (Mac2bp) and fucosylated haptoglobin (Fuc-Hpt) (a glycoprotein which is undetectable in non-fibrotic liver) were also studied as potential markers for NASH in a prediction model that demonstrated an AUROC of 0.854 on a training cohort of 124 biopsy-proven NAFLD, and of 0.844 on a validation cohort of 382 patients [64].

Recently, a large multi-analysis on 122 studies on non-invasive tests for NASH vs. NAFL has evaluated the pooled Se and Sp of every single marker proposed. None of these showed both Se or Sp >80\% [65]. In particularly, it has been evaluated the pooled Se and Sp of ALT on 8 studies (63.5\%; 74.4\%), AST on 5 studies (76.9\%; 61.9\%), IL-6 on 3 studies (60.6\%; 83.9\%), adiponectin on 4 studies (72\%; 73\%), CK18 -M30 on 15 studies (68.4\%; 74.2\%), CK18-M60 on 5 studies (73.2\%; 73.7\%), and Mac2bp on 2 studies (67\%; 79\%) [65]. Based on these findings, the authors concluded that none of these single markers or scoring systems can be recommended to differentiate NASH from NAFL [65]. Furthermore, in a recent editorial, it was pointed out that, more than the identification of NASH, the assessment of the degree of fibrosis has the greatest clinical relevance on NAFLD patients morbidity and mortality [66]. As a matter of fact, in a meta-analysis on 5 studies, with 1, 495 NAFLD patients, the presence of fibrosis in simple steatosis was associated with a greater risk of all-cause and liver-related mortality compared to NASH without fibrosis [67], and liver fibrosis dictates the long-term course of NAFLD [68]. Moreover, recent data suggest that non-invasive serum biomarkers (such as NFS) can predict mortality and CVD risk [69, 70]. Given that NAFLD/NASH "pandemic" is fueling the upsurge in CVD [71], a growing number of patients with advanced liver fibrosis will be candidates for cardiovascular therapy in the near future [72]. In this picture, non-invasive diagnostic tools could be useful in the management of NAFLD patients for stratifying liver fibrosis as well as cardiovascular risk.

In Table 1 we report all the tests aimed at NASH diagnosis together with their diagnostic accuracy.

Table 1. Summary of biomarkers for NASH diagnosis in patients with NAFLD

\begin{tabular}{|c|c|c|c|c|c|}
\hline Parameters measures & $\begin{array}{l}\text { Non-invasive test } \\
\text { abbreviation }\end{array}$ & AUROC & Sensitivity & Specificity & $\begin{array}{l}\text { Study population, } \\
\text { reference }\end{array}$ \\
\hline ALT & & 0.62 & $72 \%$ & $51 \%$ & 222 NAFLD, [39] \\
\hline \multirow{2}{*}{$\begin{array}{l}\text { ALT, insulin resistance, } \\
\text { hypertension }\end{array}$} & HAIR & 0.9 & $80 \%$ & $89 \%$ & 105 Bsp, [45] \\
\hline & & 0.68 & $57 \%$ & $77 \%$ & 213 T2DM, [46] \\
\hline $\begin{array}{l}\text { Hypertension, T2DM, AST, ALT, } \\
\text { OSAS, nonblack race }\end{array}$ & $\begin{array}{l}\text { NASH Clinical Scoring } \\
\text { System for Morbid Obesity }\end{array}$ & 0.8 & & & 200 Bsp, $[33,57]$ \\
\hline
\end{tabular}


Table 1. Summary of biomarkers for NASH diagnosis in patients with NAFLD (continued)

\begin{tabular}{|c|c|c|c|c|c|}
\hline Parameters measures & $\begin{array}{l}\text { Non-invasive test } \\
\text { abbreviation }\end{array}$ & AUROC & Sensitivity & Specificity & $\begin{array}{l}\text { Study population, } \\
\text { reference }\end{array}$ \\
\hline T2DM, OSAS, ALT, TG & & 0.79 & & & 253 Bsp, [44] \\
\hline \multirow{3}{*}{$\begin{array}{l}\text { Age, sex, weight, height, } \\
\text { TG, cholesterol, alfa2- } \\
\text { macroglobulin, ApoA1, } \\
\text { haptoglobin, total bilirubin, } \\
\text { GGT, ALT, AST }\end{array}$} & NASH test & 0.79 & $33 \%$ & $94 \%$ & $\begin{array}{l}257 \text { NAFLD + } 383 \\
\mathrm{cp},[48]\end{array}$ \\
\hline & & 0.74 & $14.1 \%$ & $95.8 \%$ & 494 obese, [49] \\
\hline & & 0.69 & $71 \%$ & $60 \%$ & 222 T2DM, [51] \\
\hline \multirow[t]{2}{*}{ IL-6 } & & 0.92 & $58 \%$ & $100 \%$ & 83 NAFLD, [53] \\
\hline & & 0.72 & $63 \%$ & $80 \%$ & 79 NAFLD, [54] \\
\hline Adiponectin, ghrelin, leptin & & 0.78 & $81.8 \%$ & $76.1 \%$ & 82 NAFLD, [55] \\
\hline \multirow[t]{2}{*}{ CK18-M30 } & & 0.83 & & & 139 NAFLD, [58] \\
\hline & & 0.83 & $66 \%$ & $82 \%$ & 822 NAFLD, [56] \\
\hline $\begin{array}{l}\text { CK18 fragments (M30 and M65 } \\
\text { pooled) }\end{array}$ & & 0.82 & $78 \%$ & $87 \%$ & $\begin{array}{l}9 \text { studies (856 } \\
\text { NAFLD), [57] }\end{array}$ \\
\hline IL-6, CK18-M65, adiponectin & & 0.9 & $84 \%$ & $85 \%$ & 79 NAFLD, [54] \\
\hline $\begin{array}{l}\text { T2DM, sex, BMI, TG, } \\
\text { CK18-M30, CK18-M65 }\end{array}$ & & 0.81 & & & 79 NAFLD, [59] \\
\hline ALT, PLT, CK18-M30 & & 0.92 & $89 \%$ & $86 \%$ & 95 NAFLD, [61] \\
\hline $\begin{array}{l}\text { CK18-M30, ALT, presence of } \\
\text { MS }\end{array}$ & Nice model & $0.88-0.83$ & & & 454 NAFLD, [60] \\
\hline $\begin{array}{l}\text { CK18-M30, CK18-M65, } \\
\text { adiponectin, resistin }\end{array}$ & NASH diagnostics & 0.91 & $96 \%$ & $70 \%$ & 101 NAFLD, [62] \\
\hline Golgi protein 73, CK18-M30 & G-NASH & 0.84 & & & 345 NAFLD, [63] \\
\hline Mac-2bp, Fuc-HPT & & 0.84 & $71.4 \%$ & $82.3 \%$ & $\begin{array}{l}124+382 \text { NAFLD, } \\
{[64]}\end{array}$ \\
\hline PIIINP & & $0.85-0.87$ & & & 172 NAFLD, [91] \\
\hline $\begin{array}{l}\text { Type IV collagen S, ferritin, } \\
\text { fasting insulin }\end{array}$ & NAFIC score & $0.78-0.85$ & & & $\begin{array}{l}177+442 \text { NAFLD, } \\
{[99]}\end{array}$ \\
\hline Type IV collagen S, AST & CA Index & $0.85-0.76$ & & & $\begin{array}{l}94+62 \text { NAFLD, } \\
{[98]}\end{array}$ \\
\hline \multirow[t]{2}{*}{ PNPLA3, AST, fasting insulin } & NASH Score & 0.77 & $71.6 \%$ & $73.5 \%$ & 296 Bsp \\
\hline & & 0.76 & $39.1 \%$ & $89.1 \%$ & 380 NAFLD, [144] \\
\hline $\begin{array}{l}\text { PNPLA3, AST, fasting insulin, } \\
5 \text { metabolites (Glu, Ile, Gly, } \\
\text { Tyr, Ser) } 3 \text { lipides (PE, TG, } \\
\text { LysoPC) }\end{array}$ & NASH ClinLipMet Score & 0.86 & $85.5 \%$ & $72.1 \%$ & 318 NAFLD, [143] \\
\hline $\begin{array}{l}\text { Panel of } 20 \text { TG (lipidomic } \\
\text { profile) }\end{array}$ & OWliver Test & 0.81 & $73 \%$ & $80 \%$ & 467 NAFLD, [150] \\
\hline Pyroglutamate & & 0.88 & $72 \%$ & $85 \%$ & $\begin{array}{l}21 \mathrm{NASH}, 38 \mathrm{NAFL} \text {, } \\
31 \mathrm{cp},[148]\end{array}$ \\
\hline \multirow[t]{3}{*}{ N-glycan (NGA2F, NA2) } & GlycoNashTest & 0.74 & & & $\begin{array}{l}47 \text { NAFLD + } 13 \mathrm{cp} \\
\text { [152] }\end{array}$ \\
\hline & & $0.75-0.66$ & & & $\begin{array}{l}51 \text { Bsp + } 224 \\
\text { NAFLD, [153] }\end{array}$ \\
\hline & & 0.72 & & & $\begin{array}{l}51 \text { Pediatric } \\
\text { NAFLD, [154] }\end{array}$ \\
\hline \multirow[t]{2}{*}{ miR-34a } & & 0.81 & & & 111 NAFLD, [164] \\
\hline & & 0.78 & & & $\begin{array}{l}6 \text { studies (406 } \\
\text { NAFLD), [165] }\end{array}$ \\
\hline
\end{tabular}

ApoA1: apolipoprotein A1; Bsp: bariatric surgery patients; cp: control patients; Glu: glutamate; Gly: glycine; lle: isoleucine; LysoPC: Iysophosphatidylcholine; miR: micro RNA; OSAS: obstructive sleep apnea syndrome; PE: phosphatidylethanolamine; PIIINP: N-terminal type III collagen pro-peptide; Ser: serine; Tyr: tyrosine; PNPLA3: patatin-like phospholipase domain containing 3 protein 


\section{Biochemical tests for evaluation of fibrosis}

Several simple scoring systems based on standard biochemical and hematological parameters were proposed for the discrimination of AF which, in NAFLD patients, is generally considered as a grade fibrosis $\geq 3$ according to Brunt or METAVIR scoring systems. AST-platelet ratio index (APRI) and FIB-4, initially proposed on HCV patients, have been subsequently validated also in NAFLD patients, showing similar reliability [73]. In particular, FIB-4, evaluating age, AST, ALT, and platelet count, is one of the best performing tests for NAFLD and showed an $80 \%$ PPV and 90\% NPV for AF, with a cutoff score $>3.25$ and $<1.3$ respectively [74]. NFS is a non-invasive test specific to fatty liver, calculated using age, BMI, diagnosis of impaired fasting glucose or diabetes, AST/ALT ratio, albumin serum levels and PLT count, that showed a PPV of $90 \%$ in detecting a liver fibrosis $>$ F3 with a cutoff score $>0.675$ and an NPV of 93\% in excluding AF with a cutoff score $<-1.455$ [75].

In a recent meta-analysis on 64 studies, which included 13, 046 NAFLD patients, APRI, FIB-4, NFS, and BARD scores (calculated using BMI, AST, and ALT) [76] were compared in diagnostic performance of AF. NFS and FIB-4 demonstrated the highest accuracy for ruling out AF with an NPV $>90 \%$ and AUROC of 0.84 for both [77], showing clinical usefulness to exclude AF [78]. Also in diabetic patients, FIB-4 and NFS have an acceptable clinical utility in excluding AF, using the standard cutoff of $<1.3$ and $<-1.455$, respectively [79]. Despite NFS and FIB-4 demonstrated to be accurate enough to be useful as first-line tools to identify patients with $\mathrm{AF}$, about $30 \%$ of patients fell into the intermediate-risk category, in which further analyses are needed to clarify the diagnosis [80]. Moreover, in patients aged $>65$ years, FIB-4 and NFS underperformed, showing unacceptable specificity. For this reason, in a recent study on 634 biopsy-proven NAFLD patients, new thresholds (FIB- $4>2$ and NFS $>0.12$ ) were proposed, in order to lower the false positive rate maintaining the same specificity [81]. Similarly, in young adults ( $<35$ years) NFS and FIB-4 showed a poor diagnostic performance, with AUROCs of $<0.53$ and, then, further investigations are needed to define an appropriate cutoff for this category [82]. In addition, it has to be pointed out that the use of these tests in a primary care referral setting, allows discriminating only patients with liver fibrosis $>$ F3. Therefore, it does not identify F2 patients, who have a lower but not negligible risk of mortality. For this reason, a cutoff of 1 has been also proposed for FIB-4, to rule out the presence of any degree of fibrosis (i.e. F0 vs. F1-4) with an AUROC of 0.843 [83].

Recently the "HEPAmet" fibrosis scoring system has been proposed, composed of clinical variables and serum markers (age, female sex, diabetes, glucose, insulin, HOMA, AST, albumin, PLT). In its validation study on 2, 452 NAFLD patients, it showed a better diagnostic performance for diagnosis of AF compared to NFS and FIB-4 (AUROC 0.85 vs. 0.80) with an Sp of 97.2\%, Se of 74\%, NPV of $82 \%$ and a PPV of 76.3\% [84].

High diagnostic accuracy in AF identification was demonstrated by FibroMeter®, a patented panel (AST, ALT, ferritin, platelet body weight, and age) that showed an AUROC 0.94, with Se 78.9\% and an Sp of 95\% [85]. Recently, a sequential combination of non-invasive test, combining FibroMeter and TE, namely Fibrometer VCTE, has been proposed in order to identify advanced liver fibrosis in patients inside the intermediate-risk grey-zone between the cutoffs of NFS and FIB-4, showing a good performance with an AUROC of 0.86 [86].

Besides the tests based on indirect marker of fibrosis, other indirect methods of assessing fibrogenic activity are based on the evaluation of some components of extracellular matrix turnover, that replace hepatocytes when the ongoing liver injury exceeds the hepatic regeneration.

The FibroTest $₫$ is a commercially available panel, previously mentioned, and showed a good diagnostic performance for AF in NAFLD patients with an AUROC of 0.84 for F3-4 [87].

Moreover, a recent study on 1, 079 NAFLD patients with a median follow-up of 6 years, demonstrated that FT provides a good long-term prognostic value for survival without liver-related deaths, with an AUROC of 0.941. In this way, FT may be useful as a second-line analysis in order to rule out those subjects at low risk from further immediate evaluation [88, 89].

Different tests include hyaluronic acid (HA) as marker of fibrosis, because it is synthesized by stellate cells and metabolized by sinusoidal endothelial cells. "Hepascore", combining HA and alfa2-macroglobulin with clinical variables (age, gender) and blood-based parameter (GGT, bilirubin, ALT, AST), showed a good 
performance to identify AF in NAFLD patients with an AUROC of 0.81 and an NPV of $92 \%$, but a better diagnostic performance in chronic viral hepatitis [90].

The ELF score is a commercial panel that evaluates, in addition to HA, tissue inhibitor of matrix metalloproteinase 1 (TIMP-1), and PIIINP, which, just as a single biomarker, provided a good reliability for diagnosis of cirrhosis with NPV of 95\% [91]. The ELF score demonstrated a good performance with an AUROC of 0.9 using the threshold of 0.35 . However, in a recent meta-analysis on 11 studies, it was found to have a limited specificity in excluding AF in NAFLD patients in the context of low disease prevalence (5-10\%), suggesting a re-evaluation of its threshold values $[92,93]$.

Recently, a new serum biomarker derived from collagen III synthesis, type III collagen formation (Pro-C3), was proposed in a study on 150 biopsy-proven NAFLD patients, showing an AUROC of 0.91 with an NPV of $97 \%$ for AF identification. Subsequently, it was included in a score incorporating clinical variables, the ADAPT (age, diabetes, and platelet), and studied in a cohort of 449 patients, confirming higher diagnostic accuracy (AUROC 0.87) compared to APRI, FIB-4 and NFS [94, 95].

Similarly, on a cohort of 396 NAFLD patients, an algorithm using TIMP-1, HA and alfa-2 macroglobulin was recently validated, showing an AUROC 0.86 for identification of AF [96].

Type IV collagen-7S is another fibrosis marker studied for the detection of NASH and, recently, AF. It was evaluated as a single marker of liver fibrosis, showing a good diagnostic performance with AUROC of 0.827 and NPV of 0.84 [97], but also included in two predictive models, the NAFIC score (ferritin, fasting insulin, collagen IV) and CA-fibro index (collagen IV and AST) with AUROC of 0.824 and 0.845 respectively $[98,99]$.

Finally, a recent study showed the diagnostic accuracy of collagen IV and Mac2bp in the detection of AF in NAFLD, reporting an AUROC of 0.83 for both, but further investigations are needed for their use in clinical practice [100].

In Table 2 tests aimed at the evaluation of fibrosis in NAFLD together with their diagnostic accuracy were reported.

Table 2. Summary of biochemical tests for AF in patients with NAFLD

\begin{tabular}{|c|c|c|c|c|c|}
\hline Parameters Measures & $\begin{array}{l}\text { Non-invasive } \\
\text { test }\end{array}$ & AUROC & Cutoff & $\begin{array}{l}\text { Diagnostic } \\
\text { accuracy }(\%)\end{array}$ & Study population, reference \\
\hline \multirow[t]{4}{*}{ AST, PLT } & APRI score & 0.76 & $>1$ & Se 66-Sp 91, NPV 87 & 245 NAFLD, [85] \\
\hline & & 0.77 & $<1$ & Se $50-S p 84$ & 6, 877 NAFLD, [77] \\
\hline & & & $>1.5$ & Se 18.3-Sp 96.1 & \\
\hline & & 0.86 & 0.42 & Se 84-Sp 75, NPV 96 & 213 T2DM, [46] \\
\hline \multirow[t]{7}{*}{ Age, AST, ALT, PLT } & FIB-4 index & 0.8 & $<1.3$ & Se 74-Sp 71 & 541 NAFLD, [74] \\
\hline & & & $>2.67$ & Se 33-Sp 98 & \\
\hline & & 0.84 & $>2.67$ & Se 26.6-Sp 96.5 & 8, 635 NAFLD, [77] \\
\hline & & & $>3.25$ & Se 31.8-Sp 96 & \\
\hline & & 0.72 & $>2$ & Se 77-Sp 70 & $\begin{array}{l}61 \geq 65 \text { years NAFLD, [81] (reduced } \\
\text { false positive in age }>65 \text { years) }\end{array}$ \\
\hline & & 0.84 & $<1$ & $\begin{array}{l}\text { Se } 100-S p 94.3 \text { (F0 } \\
\text { vs. F1-4) }\end{array}$ & $\begin{array}{l}108+239 \text { NAFLD, [83] (excluding } \\
\text { any fibrosis) }\end{array}$ \\
\hline & & 0.78 & 1.6 & Se 68-Sp 75, NPV 93 & 213 T2DM, [46] \\
\hline \multirow{6}{*}{$\begin{array}{l}\text { AGE, BMI, albumin, } \\
\text { AST/ALT ratio, } \\
\text { hyperglycemia, PLT }\end{array}$} & NFS & 0.84 & $>0.67$ & PPV 90 & 733 NAFLD, [75] \\
\hline & & & $<-1.45$ & NPV 93 & \\
\hline & & 0.84 & $<-1.45$ & Se 72-Sp 70 & 9, 392 NAFLD, [77] \\
\hline & & 0.81 & $<-1.45$ & Se 93-Sp 20 & $\begin{array}{l}61 \geq 65 \text { years NAFLD, [81] (reduced } \\
\text { false positive in }>65 \text { years) }\end{array}$ \\
\hline & & 0.64 & $<-1.45$ & Se $91-S p 40$ & 69 T2DM, [46] \\
\hline & & & -0.053 & Se 68-Sp 55, NPV 90 & \\
\hline
\end{tabular}


Table 2. Summary of biochemical tests for AF in patients with NAFLD (continued)

\begin{tabular}{|c|c|c|c|c|c|}
\hline Parameters Measures & $\begin{array}{l}\text { Non-invasive } \\
\text { test }\end{array}$ & AUROC & Cutoff & $\begin{array}{l}\text { Diagnostic } \\
\text { accuracy }(\%)\end{array}$ & Study population, reference \\
\hline \multirow{2}{*}{$\begin{array}{l}\text { BMI, T2DM, AST/ALT } \\
\text { ratio }\end{array}$} & \multirow[t]{2}{*}{ BARD score } & 0.81 & $2-4$ & NPV 96 & 827 NAFLD, [76] \\
\hline & & 0.76 & $<2$ & Se 76-Sp 61 & 7, 791 NAFLD, [77] \\
\hline \multirow{2}{*}{$\begin{array}{l}\text { Age, weight, glucose, } \\
\text { AST, ALT, ferritin, PLT }\end{array}$} & \multirow[t]{2}{*}{ FibroMeter } & 0.94 & $>0.71$ & Se 79-Sp 96, NPV 92 & 235 NAFLD, [85] \\
\hline & & 0.81 & & $\begin{array}{l}\text { Se } 88-S p \text { 93, NPV } \\
92.7\end{array}$ & 938 NAFLD, [86] \\
\hline $\begin{array}{l}\text { Age, weight, glucose, } \\
\text { AST, ALT, ferritin, PLT, } \\
\text { VCTE }\end{array}$ & $\begin{array}{l}\text { Fibrometer } \\
\text { VCTE }\end{array}$ & 0.86 & $0.32-0.69$ & $\begin{array}{l}\text { Se } 85-S p \text { 92, NPV } \\
90.7\end{array}$ & 938 NAFLD, [86] \\
\hline \multirow{4}{*}{$\begin{array}{l}\text { GGT, bilirubin, } \\
\text { haptoglobin, ApoA1, } \\
\text { alfa2 macroglobulin }\end{array}$} & \multirow[t]{4}{*}{ FibroTest@ } & 0.81 & $<0.3$ & NPV 90 & 170 NAFLD + 954 cp, [47] \\
\hline & & 0.84 & $>0.7$ & PPV 73 & $\begin{array}{l}267 \text { NAFLD, } 429 \text { ALD, } 724 \text { mixed, } \\
\text { [87] }\end{array}$ \\
\hline & & 0.85 & $<0.27$ & & 494 obese, [49] \\
\hline & & 0.7 & 0.35 & Se 64-Sp 74 NPV 92 & 130 T2DM, [46] \\
\hline $\begin{array}{l}\text { Age, sex, bilirubin, } \\
\text { GGT, alfa2- } \\
\text { macroglobulin, HA }\end{array}$ & Hepascore & 0.81 & $>0.37$ & Se 75-Sp 84, NPV 92 & 242 NAFLD, [90] \\
\hline \multirow[t]{2}{*}{ HA, PIIINP, TIMP-1 } & \multirow[t]{2}{*}{ ELF test } & 0.9 & $>0.35$ & Se 80-Sp 90, NPV 94 & 196 NAFLD, [92] \\
\hline & & 0.83 & $<10.18$ & Sp 90 & 11 studies (4, 452 NAFLD), [93] \\
\hline $\begin{array}{l}\text { Age, T2DM, PLT, } \\
\text { Pro-C3 }\end{array}$ & ADAPT & 0.87 & $>6.32$ & PPV 48.4-NPV 96.6 & 150 + 281 NAFLD, [94] \\
\hline \multirow[t]{2}{*}{ Age, BMI, PLT, T2DM } & \multirow[t]{4}{*}{$A B C 3 D$} & 0.88 & $>3$ & Se 77-Sp 82, NPV 84 & 151 discovery cohort NAFLD, [95] \\
\hline & & 0.81 & & Se 66-Sp 75, NPV 80 & 298 validation cohort NAFLD, [95] \\
\hline $\begin{array}{l}\text { TIMP-1, HA, alfa2 } \\
\text { macroglobulin }\end{array}$ & & 0.85 & 17 & Se 79-Sp 75, NPV 92 & 396 NAFLD, [96] \\
\hline Type IV collagen S & & 0.82 & $>177$ & Se 77-Sp 84, NPV 84 & 148 NAFLD, [97] \\
\hline $\begin{array}{l}\text { Type IV collagen S, } \\
\text { AST }\end{array}$ & CA index & $0.84-0.91$ & & & 94 + 62 NAFLD, [98] \\
\hline \multirow{2}{*}{$\begin{array}{l}\text { Mac-2bp, Type IV } \\
\text { collagen S }\end{array}$} & & \multirow[t]{2}{*}{0.83} & $>0.83$ & Se 91-Sp 61, NPV 87 & 165 NAFLD, [100] \\
\hline & & & $>5.2$ & & \\
\hline $\begin{array}{l}10 \text { urinary steroid } \\
\text { metabolites }\end{array}$ & $\begin{array}{l}\text { GMLVQ } \\
\text { analysis }\end{array}$ & $0.99-1$ & & & 275 NAFLD, [147] \\
\hline $\begin{array}{l}\text { SNP IFNL 4, HOMA-IR, } \\
\text { GGT, AST, ALT, PLT }\end{array}$ & $\begin{array}{l}\text { FibroGENE } \\
\text { DT }\end{array}$ & 0.8 & & NPV 96 & 488 NAFLD, [158] \\
\hline GUT microbiome model & & 0.936 & & & 86 NAFLD, [159] \\
\hline Inc RNAs + FIB-4 or TE & & 0.89 & & & 88 + 50 NAFLD, [166] \\
\hline
\end{tabular}

GMLVQ: generalized matrix learning vectors quantization; IncRNAs: including long non-coding RNAs; SNP IFNL 4: single nucleotide polymorphism interferon lamba 4; VCTE: vibration controlled transient elastography

\section{Imaging techniques}

As mentioned above, in most of the cases liver US is the first exam that leads to the finding of hepatic steatosis. The typical US features of fatty liver are: posterior US beam attenuation, loss of echoes from the diaphragm, and loss of echoes from the walls of the portal vein [101]. Based on these features, the steatosis can also be subjectively scored as mild, moderate, and severe with a fair degree of accuracy in detection of moderatesevere fatty liver, compared to liver biopsy. This has been confirmed by a meta-analysis on 34 studies that showed a pooled sensitivity and specificity respectively of $85 \%$ and $95 \%$ [102]. Unfortunately, in clinical practice, US shows a good sensitivity only in patients with a percentage of liver fat content above 12.5-25\%. Therefore, patients with a lower, but still relevant, fat liver content may be missed [103]. Furthermore, 
its diagnostic accuracy is reduced in patients with obesity or coexistent CLD [104]. All the international guidelines consider liver US as the first-line imaging technique for the diagnosis of fatty liver in both clinical and epidemiological settings due to its safety, cost-effectiveness, and availability. Recent data suggest that US using semi-quantitative scores can detect steatosis as low as $10 \%$ [105]. A more accurate assessment of the amount of liver fat and the degree of liver fibrosis is performed by US-based or magnetic resonancebased techniques. MRI is more accurate, but its availability is very limited due to its high costs. A US-based technique, the "controlled attenuation parameter" (CAP), might be more accurate than conventional US for detecting liver steatosis, and has also the advantage of simultaneously estimating liver fibrosis, being coupled with liver stiffness measurement (LSM) [106]. However, LSM with CAP could not be readily available outside specialized centers. No data are available on a direct comparison of CAP and semi-quantitative US scores in patients with NAFLD or other CLD.

The imaging techniques aimed at the evaluation of fibrosis in NAFLD together with their diagnostic accuracy are reported in Table 3.

Table 3. Summary of imaging techniques for evaluation of liver stiffness in patients with NAFLD

NMR-based techniques

\begin{tabular}{|c|c|c|c|c|c|}
\hline & End-point & AUROC & Cutoff (kPa) & Diagnostic accuracy (\%) & Study population, reference \\
\hline \multicolumn{6}{|l|}{ 2D MRE } \\
\hline & AF & $0.93-0.96$ & $3.6-4.8$ & Se 85-Sp 85; PPV 71-NPV 93 & 628 NAFLD, $[77,113]$ \\
\hline & Cirrhosis & 0.9 & $>4.1$ & Se 86-Sp 93; PPV 53-NPV 98 & 384 NAFLD, [77] \\
\hline \multicolumn{6}{|l|}{ 3D MRE } \\
\hline$-40 \mathrm{~Hz}$ & AF & 0.98 & 2.43 & Se 100-Sp 94; PPV 72-NPV 100 & 100 NAFLD, [114] \\
\hline$-60 \mathrm{~Hz}$ & & 0.92 & 3.4 & & \\
\hline$-40 \mathrm{~Hz}$ & Cirrhosis & 0.99 & 3.2 & Se 86-Sp 96; PPV 81-NPV 97 & \\
\hline$-60 \mathrm{~Hz}$ & & 0.98 & 4 & & \\
\hline
\end{tabular}

US-based techniques

\begin{tabular}{|c|c|c|c|c|c|}
\hline & End-point & AUROC & Cutoff (kPa) & Diagnostic accuracy (\%) & Study population, reference \\
\hline \multicolumn{6}{|l|}{ TE (Fibroscan®) } \\
\hline - M Probe & AF & 0.87 & $7.6-8$ & Se 89-Sp 77; PPV 43-NPV 95 & 1, 540 NAFLD, [77] \\
\hline - XL Probe & & 0.86 & $5.7-9.3$ & Se 75-Sp 74; PPV 43-NPV 95 & 579 NAFLD, [77] \\
\hline - M Probe & Cirrhosis & 0.92 & $10.3-11.3$ & Se 87-Sp 86; PPV 46-NPV 98 & 1, 362 NAFLD, [77] \\
\hline - XL Probe & & 0.94 & $7.2-16$ & Se 84-Sp 80; PPV 39-NPV 98 & 654 NAFLD, [77] \\
\hline \multicolumn{6}{|l|}{ ARFI } \\
\hline & SF & 0.89 & & Se $80-S p 85$ & 723 NAFLD, [136] \\
\hline \multicolumn{6}{|l|}{ 2D-SWE } \\
\hline & SF & 0.85 & 9.2 & & 156 NAFLD, [138] \\
\hline & AF & 0.89 & $>8.3<10.7$ & Se 71-Sp 90 & 291 NAFLD, [139] \\
\hline & Cirrhosis & 0.91 & 13.5 & & 156 NAFLD, [138] \\
\hline & & 0.88 & $>10.5$ & Se $90-S p 72$ & 291 NAFLD, [139] \\
\hline
\end{tabular}

AF (F3); Cirrhosis (F4); significant fibrosis: $4>F \geq 2$

\section{MRI}

Magnetic resonance is the most accurate non-invasive technique for the evaluation of hepatic steatosis, because it can directly quantify the amount of liver TG through magnetic resonance spectroscopy (MRS) or 
magnetic resonance imaging (MRI), by quantifying the proton density fat fraction (PDFF). MRS-PDFF, due to various technical limitations (need for expertise in protocol prescription, data collection, and spectral analysis) and the lack of spectroscopy software in routine scanners, is not commonly used, while MRI-PDFF is more routinely available [107]. PDFF is represented by the fraction of MRI-visible protons bound to fat, divided by all protons in the liver (bound to fat and water). It allows a quantitative and objective evaluation of the entire fat content in the liver and can detect as little as 3\% of steatosis [108].

The accuracy of MRI-PDFF was compared to liver histology by a multi-center study reporting a high AUROC (0.95) for liver steatosis identification [109]. Moreover, as reported by single-center studies, the MRI-PDFF seems to be more sensitive than liver histology in the longitudinal assessment of liver steatosis changes over time [110]. In a secondary analysis of the multi-center phase II trial on 113 patients enrolled to obeticholic acid or placebo, before and after the 72 weeks of treatment, steatosis was measured by MRI-PDFF paired with liver histology, and showed an accurate concordance between the decline $>30 \%$ of MRI-PDFF and $>2$ point of NAFLD activity score, defined as significant improvement of liver steatosis [111]. MRI-PDFF is less susceptible to sampling errors compared to liver biopsy, because it detects the total amount of TG in the whole liver, but it cannot give information on necroinflammation. In fact, even if some studies have attempted to evaluate the ability of MR-based or US-based techniques to discriminate simple steatosis from $\mathrm{NASH}$, at the moment neither of them can reliably be used for this purpose [112].

On the contrary, fibrosis may be non-invasively detected by using other techniques, of which "stiffness" (or "elasticity") and its family of related parameters are the best validated on the liver. The collagen deposition associated with fibrosis confers parenchymal rigidity, which can be evaluated by assessing its stiffness. The MRE determines the liver stiffness through the analysis of microdisplacements ("shear waves") of the tissue, using a modified phase-contrast imaging sequence able to detect the propagation of the shear wave within the hepatic parenchyma [113]. The shear wave's velocity is converted in LSM that is expressed, as a final result, in meters per second or $\mathrm{kPa}$. In a meta-analysis on 9 studies on a total of 232 biopsy-proven NAFLD patients, high accuracy of MRE to detect AF and cirrhosis (AUROC 0.93 and 0.92 respectively) was reported, with an optimal cutoff for AF of $3.64 \mathrm{kPa}$ [113]. In another recent meta-analysis on 5 studies (628 NAFLD patients) an AUROC of 0.96 for AF detection has been estimated using the same cutoff value [77]. A further improvement of the diagnostic accuracy of the MRE has been proposed by the use of 3D technology. In fact, in a head-to-head comparison with 2D-MRE, 3D-MRE showed an AUROC of 0.96 for AF detection [114]. However, 3D-MRE is a meticulous and time-consuming exam and, among other things, it has yet to be validated through multicenter studies. MRE has a low failure rate (1-2\%) and its failure is associated with massive ascites, iron deposition, or high BMI [115]. The effect of BMI is still debated, in fact, a recent study on 111 patients with a mean BMI of $40.3 \mathrm{~kg} / \mathrm{m}^{2}$, the intra-observer agreement was higher with MRE than with biopsy, providing an AUROC of 0.93 for detection of AF[116].

A novel MR-based method is the multiparametric MRI (Liver-MultiScan), that proposes to measure liver steatosis and correlate it not only with fibrosis, but also with inflammation, using T1 mapping for fibrosis and inflammation, T2 mapping for liver iron quantification and MRS for liver fat quantification [117]. In a pilot proof-of-concept study on 71 patients, multiparametric MRI showed an AUROC of 0.83 for detection of hepatocyte ballooning and lobular inflammation [118]. Other interesting data on these topics derive from a preclinical study on pigs and mice, in which the addition of damping ratio to 2D-MRE and MRI-PDFF contributes to higher diagnostic accuracy for detection of both inflammation and fibrosis at early stages, even before the development of histological alterations [119]. Nevertheless, these promising results still need further investigations.

\section{US-based imagining technique}

$\mathrm{TE}$

Unlike MRI, the US-based elastography detect the velocity of shear wave induced by US on liver parenchyma in order to estimate the liver stiffness as indirect marker of fibrosis. The pioneer US-based technique is the vibrant-controlled TE, developed using a dedicated device (Fibroscan $囚$ ) [120]. Initially validated in viral hepatitis patients, TE has confirmed high diagnostic accuracy in evaluating liver fibrosis also in NAFLD. In a 
meta-analysis on TE studies (1, 047 NAFLD patients) performed until 2013, it reported an AUROC of 0.760.98 for detection of Metavir-F3 and an AUROC of 0.91-0.99 for F4, respectively at the cutoff of 8-10.4 kPa and $>10.3 \mathrm{kPa}$ [56]. Worse diagnostic accuracy was reported for the detection of F2 (AUROC 0.79-0.87). TE is limited by the ascites or severe obesity, as the interposition of fluids or fat between the chest wall and the liver prevents the correct determination of the shear waves. A lower rate of failure or unreliable result is determined by the use of the XL-probe, providing, as shown in a most recent meta-analysis on 19 studies (4 using XL-probe), similar diagnostic accuracy in detection of AF compared to the M-probe (AUROC 0.87 vs. 0.86) [77]. Nevertheless, it must be noted that liver stiffness may be overestimated by TE in case of high liver inflammation activity (transaminase flare), extrahepatic cholestasis, or congestive heart failure [121].

Above all, compared to other routinely available biomarker of AF (FIB-4, APRI, NFS, BARD), TE shows the highest NPV, allowing confidently excluding AF at a cutoff < $8 \mathrm{kPa}$ in NAFLD patients (NPV 95-100\%) [122]. Moreover, compared to the other tests, only the FibroMeter has not been shown to be less accurate than $\mathrm{TE}$, and, as previously mentioned, a non-invasive test based on the combination of these two tools, namely Fibrometer VCTE, has been proposed [86, 123]. Recently, TE has been evaluated in combination with other serum biomarkers, improving the non-invasive detection of hepatic fibrosis in patients with NAFLD. The use in serial combination of TE, NFS and FIB-4 in patients in the grey area of the first test or in those with high values of liver median stiffness $(>9.6 \mathrm{kPa})$ or low NFS or FIB-4 value $(<-1.45$ and $<1.3$ respectively), increased the diagnostic performance and reduced the diagnostic uncertainty area compared to the use of these tests alone [122]. The combination of ELF test and TE has shown higher diagnostic accuracy for the diagnosis of AF (Sp 97.9\%) compared to ELF test alone (Sp 90.6\%) [124]. The FibroScan-AST (FAST) score is a novel model (composed of Liver Median Stiffness, controlled attenuated parameter and AST), that showed a good diagnostic performance in identifying NASH with a Nash Activity Score $>4+$ F $>2$ with a PPV of 0.830.81 and an NPV of $0.85-0.71$, respectively in derivation and validation cohort [125].

Although some comparison studies on the diagnostic accuracy of MRE and TE did not show a static difference between the two techniques, a recent meta-analysis, on 230 biopsy-proven NAFLD, demonstrated an undoubted diagnostic superiority of MRE compared to TE in detection of each stage of fibrosis [126].

CAP is a novel technique for the evaluation of hepatic steatosis. Using the TE M or XL-probes, it estimates the amount of liver fat by measuring the degree of US attenuation exerted by hepatic fat, expressed as decibel per meter $(\mathrm{dB} / \mathrm{m})$ [127]. Despite the fact that the initial study described good accuracy in detection of steatosis (AUROC of $0.91,0.95$ and 0.89 for steatosis $\geq 11 \%, \geq 33 \%$ and $\geq 66 \%$ respectively) [127], several subsequent studies reported lower AUROCs (0.79, 0.76 and 0.76), at the limits of statistical significance, suggesting a limitation in precisely discriminating adjacent degrees of steatosis $[128,129]$. A recent meta-analysis on 2 , 735 patients (537 NAFLD) reported, for steatosis degrees of $\geq 11 \%, \geq 33 \%$ and $\geq 66 \%$, AUROCs of $0.82,0.86$ and 0.88 at the proposed cutoff values of $248 \mathrm{~dB} / \mathrm{m}, 268 \mathrm{~dB} / \mathrm{m}$, and $280 \mathrm{~dB} / \mathrm{m}$ respectively [130]. As TE, CAP is influenced by BMI, but comparison studies between $\mathrm{M}$ - and XL-probes reported conflicting results about the cutoff values $[131,132]$. In comparison with MRI-PDFF, CAP underperformed for the diagnosis of all grades of steatosis (AUROC 0.99 vs. 0.85) [133].

At the moment, the proposed cutoff values for $\geq \mathrm{S} 2$ steatosis range from $280 \mathrm{~dB} / \mathrm{m}$ to $310 \mathrm{~dB} / \mathrm{m}$ with an NPV of about 70\%, therefore further studies are necessary before any firm conclusion can be drawn [112].

Acoustic radiation force imaging

Acoustic radiation force imaging (ARFI) elastography is a US-based technique that uses the point shear wave elastography (pSWE). Compared to TE, pSWE has the vantage to be integrated into conventional US systems, evaluating the velocity of shear waves induced by a single acoustic impulse in a small region of interest (ROI) [134]. In CLD, ARFI has demonstrated good diagnostic accuracy for detection of AF (AUROC 0.84 ) and cirrhosis (AUROC 0.91), as reported in a systematic meta-analysis on 36 studies, but few studies were conducted on NAFLD patients [135]. In a review on 7 studies (723 NAFLD patients), a summary AUROC of 0.89 for detection of significant fibrosis ( $4>\mathrm{F} \geq 2$ ) was reported, with a summary sensitivity and specificity of $80 \%$ and $85 \%$ respectively [136]. However, at the moment, data on diagnostic accuracy of AF and cirrhosis are not still available and, therefore, pSWE is not included in the current guidelines of NAFLD. In a recent study 
of comparison between MRE and ARFI on a cohort of 125 biopsy-proven NAFLD patients, high diagnostic accuracy of MRE for diagnosis of any fibrosis was reported, especially in obese patients [137].

\section{D-SWE}

2D-SWE is the most novel US-based elastography technique and, it evaluates the velocity of shear waves induced by multiple acoustic impulses in a larger ROI $(2 \mathrm{~cm} \times 2 \mathrm{~cm})$, as single image or in real-time [134]. As ARFI, it is integrated into conventional ultrasonography systems. To date, few studies have evaluated the diagnostic accuracy of 2D-SWE in NAFLD patients. In a large meta-analysis on 1, 340 patients a subgroup of 156 NAFLD patients were included. In these patients, it showed an AUROC of 0.85 and 0.91 for diagnosis of significant fibrosis and cirrhosis respectively. In another subgroup of 91 NAFLD, a significantly better performance in diagnosis of AF compared to TE (AUROC difference 12\%; $P=0.003$ ) was also reported [138]. In a comparison study with TE and ARFI on 291 NAFLD, 2D-SWE outperformed for significant fibrosis and showed a similar or slightly better diagnostic accuracy for diagnosis of AF and cirrhosis. The cutoffs reported with a sensitivity and specificity $>90 \%$ were $8.3-10.7 \mathrm{kPa}$ for F3 and $>10.5 \mathrm{kPa}$ for F4 [139].

Furthermore, in a recent comparison study on 62 biopsy-proven NAFLD subjects, 2D-SWE did not demonstrate lower diagnostic accuracy than MRE for detection of AF. Nevertheless, even if this technique gave promising results, it needs further validation in NAFLD settings [140].

\section{Future directions}

Several novel strategies to detect NASH and AF in NAFLD patients are based on identification of molecules by OMICS approaches (genomic, metabolomic, proteomic, and lipidomic), providing a useful framework for designing and validating highly accurate predictive models. Circulating oxidized fatty acids and products of arachidonic acid metabolism associated with NASH were identified by a lipidomic approach [141]. An excellent NASH prediction model (AUROC 1.0) composed of a panel of plasma eicosanoids and other polyunsaturated fatty acids metabolites was identified in a proof-of-concept study [142]. Moreover, recently was proposed the "NASH ClinLipMet" Score, a novel model, based on lipid, metabolites, clinical markers, and PNPLA genotype, which identifies NASH patients with high accuracy (AUROC 0.86) [143, 144].

As regards the proteomic approach, several protein-based biomarkers were identified, by means of mass spectrometry, which were able to identify NASH and AF. In a biomarkers discovery study on 69 NAFLD patients and 19 obese controls, between 1, 700 serum proteins studied, 6 patterns of proteins expression were identified that showed significant changes between simple steatosis, NASH and AF (F3/F4), able to differentiate with high accuracy these groups [145]. Using the proteomic approach, a highly multiplexed protein assay, namely SOMAscan, was developed on a 443-patient training set, showing excellent diagnostic accuracy (AUROC 0.932) in identifying steatosis in patients carrying the PNPLA3 rs 738409 genotype [146]. A novel biomarker derived from the analysis of urinary steroid metabolome, was evaluated on 275 subjects (121 biopsy-proven NAFLD, 48 alcohol-related cirrhosis and 106 controls) [147]. This gas chromatographymass spectrometry approach demonstrated not only high accuracy in discriminating AF (AUROC 0.92), but also in distinguishing alcohol related from NAFLD related cirrhosis [147].

The metabolomics technique was used to differentiate metabolic subtypes of NAFLD. In a study on 90 patients (21 NASH, 38 simple steatosis, 31 controls), among 56 selected metabolites, pyroglutamate showed the higher accuracy in NASH identification (AUROC 0.88), also compared with tumor necrosis factor-alfa, IL-8, and adiponectin [148]. In a recent translational study, a NASH metabolic profile was induced in methionineadenosyltransferase-1a knockout mice, which spontaneously develops NASH, and the serum metabolomes were compared with those of 535 biopsy-proven NAFLD subjects, identifying a specific metabolomics profile that could distinguish NASH from simple steatosis [149].

The OWliver is a commercial test for diagnosis of NASH based on metabolomics profile of 20 metabolites and validated in a cohort of 467 NAFLD patients, demonstrating good diagnostic accuracy in discrimination of NASH from NAFL, with an AUROC of 0.81 [150]. In order to identify the bile acid metabolome in NASH, a small study on 22 subjects (7 NASH) showed an increase in taurine and glycine-conjugated primary and 
secondary bile acids, hypothesizing a role of hydrophobic and cytotoxic secondary species of these bile acids in the pathogenesis of NASH [151].

In a study on 47 biopsy-proven NASH and 13 healthy controls, the changes in glycosylation were evaluated as biomarker of liver damage. The N-glycan profile was performed and the concentration of two glycans (NGA2F and NA2) was associated with the severity of NASH [152]. The logarithm ratio of NGA2F and NA2, namely GlycoNashTest, showed good diagnostic accuracy for the detection of NASH and AF, with an AUROC of 0.74 and 0.87 respectively [152]. These results were confirmed in a subsequent validation study on 224 NAFLD patients and also in a pediatric cohort of 51 NAFLD $[153,154]$. These results demonstrated an increase in under-galactosylation of serum proteins during chronic liver inflammation, but further investigations are necessary for a clinical application of these evidences.

As far as genomics are concerned, two genetic variants, located in PNPLA3 and Transmembrane 6 superfamily member 2 (TM6SF2) hepatic stellate cells variant, were associated with an increased risk of NAFLD, but their accuracy in predicting disease is not higher compared to other noninvasive biomarkers $[155,156]$. Recently the genetic variant rs641738 C>T located in membrane bound O-acyltransferase domain-containing 7 gene (MBOAT7) was also associated with an increased risk of development and severity of NAFLD [157]. Despite the importance of the impact of these genetic variants in a complex disease like NAFLD, the effect of a single mutation is unlike to be sufficient to be clinically meaningful. In a recent study on 4, 277 patients (488 NAFLD), the diagnostic accuracy of a single nucleotide polymorphism in intronic region of interferon lambda 4, incorporated with other biomarkers (HOMA-IR, GGT, AST, ALT, PLT) of fibrosis in a model named FibroGENE DT, was evaluated for the assessment of fibrosis severity, finding an AUROC of 0.8 and 0.83 for prediction of significant fibrosis and cirrhosis respectively, with an NPV of $96 \%$ in excluding cirrhosis [158].

Another kind of approach for the assessment of NAFLD is based on metagenomics signature of GUT microbiome, by the evaluation of its composition. In a preliminary study on 86 NAFLD biopsy-proven patients, the whole GUT-microbiome genome was sequenced from stool samples, identifying 37 bacterial species that were used to construct a Random Forest classifier model with robust diagnostic accuracy for AF (AUROC 0.936) [159]. Recently, in a study on GUT-microbiome composition on 50 patients with different CLD, evaluated by Fibroscan, Prevotella copri was identified as the strongest predictive microbe for AF in NAFLD patients (AUROC 0.82) [160]. In this way, this study reported that the microbial profile of advanced CLDs is characterized by an increase in the genus of Prevotella spp and a decrease in Bacteroides, encouraging their use as non-invasive markers of liver fibrosis. Similarly, in a study on 87 NAFLD biopsy-proven children compared to 37 children without NAFLD, an abundance of Prevotella copri was also found to be associated with severe fibrosis. The study also provided a predictive model that identified F3/F4 patients with an AUROC of 0.87 and was based on the measurement of ALT together with the quantification of genes encoding flagellar biosynthesis proteins [161].

Other emerging evidences on potential NAFLD biomarkers derive from studies on circulating extracellular vesicles (EV: exosomes and ectosomes) that contain various molecules, such as proteins, microRNAs, and DNA, of which an altered expression has been demonstrated in CLDs. In a study that carried out the profile of blood EVs using flow cytometry, ectosomes were demonstrated to be increased in monocytes and natural killer T-cells and decreased in neutrophils and leuco-endothelial cells in NASH patients, thus showing that the quantification of immune cell microparticles could be a potential diagnostic strategy for differentiating NASH from simple steatosis [162].

miRNAs regulate the post-transcriptional gene expression and it has been demonstrated to contribute to NAFLD pathogenesis at various levels. miR-122 is a key regulator of hepatic fatty-acid metabolism and its levels in circulating exosomes were found upregulated in NASH in comparison to simple steatosis, correlating with histological severity [163]. miR-34a concentration was also found significantly higher in NAFLD and, in a study on 111 biopsy-proven NAFLD, demonstrated to have higher diagnostic accuracy for diagnosing NASH (AUROC 0.81) in comparison to ALT, CK-18, FIB-4, and APRI [164]. A recent meta-analysis on 37 studies evaluated the diagnostic accuracy of serum levels of miR-122 and miR-34a, showing for the discrimination 
between NAFL and NASH an AUROC of 0.82 and 0.78, respectively [165]. Recently, a combination of coding and non-coding RNA expression levels, derived from a whole transcriptome analysis, was evaluated as potential biomarkers both of the presence of NAFLD and severity of fibrosis. Good diagnostic accuracy was found for detection of AF when the expressions of coding RNA of transforming grow factor beta 2 (TGFB2)/ non-coding RNA of TGFB2-overlaping transcript 1 were associated with FIB-4 (AUROC 0.891) or TE (AUROC 0.892) [166]. These approaches are promising, but their application in clinical practice is still limited by the lack of standardized protocols.

\section{Conclusions}

At the moment, in the field of NAFLD a wide range of non-invasive serum and imaging biomarkers have been developed with the aims of discriminating patients with NASH and evaluating the degree of steatosis and liver fibrosis.

Regarding NASH identification, the single markers or scoring systems didn't demonstrate either acceptable sensitivity and specificity or sufficient validation. Thus, at the present time, noninvasive serumbiomarkers can't be widely recommended to differentiate NASH from simple steatosis. Moreover, also imaging techniques haven't demonstrated reliable diagnostic accuracy or a sufficient validation for NASH discrimination, although MR-based modalities (multiparametric MRI) are promising. Therefore, liver biopsy still remains the most complete exam for the assessment of the patient with NAFLD.

Nevertheless non-invasive tests can be very useful, particularly for screening purposes, and their choice should be tailored according to the clinical settings (primary health care or referral center) and clinical needs (screening, staging of fibrosis disease, and follow-up). NFS and FIB-4, simple and inexpensive, should be used as first-line tests in primary health-care settings, allowing ruling out patients without AF. Other patented tests as FT, FibroMeter, Hepascore, or ELF, even if more specific and with a higher PPV for detecting AF, have the inconvenience of being performed at a cost. Imaging techniques, US-based (TE, 2D-SWE, and ARFI) or MRN-based (MRE), are more suited for referral centers, with the aim of identifying the patients who require a final diagnosis by liver biopsy. MRI-PDFF seems to be the most accurate method for the detection and grading of steatosis, but its application, at the time, seems to be suited for the assessment and follow-up of patients included in clinical trials. On the contrary, CAP could be used to identify steatosis in large unselected population settings, but an optimal definition of its cutoffs has yet to be accepted by international guidelines. Even if growing evidences show that serum markers and LSM may identify NAFLD patients at high risk of liver-related complications, good, accurate and widely applicable biomarkers that allow not only a precise stratification of fibrosis, but also the monitoring of the disease progression and therapeutic response are still to be found.

\section{Abbreviations}

2D-SWE: two-dimensional shear wave elastography

AF: advanced fibrosis

ALT: alanine transaminase

ApoA1: apo-lypoprotein A1

APRI: AST-platelet ratio index

ARFI: acoustic radiation force imaging

AST: aspartate transaminase

AT: Acti-Test

AUROC: area under the receiver operating curve

Bsp: bariatric surgery patients

CAP: controlled attenuation parameter

CK18: cytokeratin 18 
CLD: chronic liver disease

cp: control patients

CVD: cardiovascular disease

ELF: enhanced liver fibrosis

EV: extracellular vesicles

FIB-4: fibrosis 4 index

FT: FibroTest

Fuc-Hpt: fucosylated haptoglobin

GGT: gammaglutamil transpeptidase

Glu: glutamate

Gly: glycine

GMLVQ: generalized matrix learning vectors quantisation

HA: hyaluronic acid

HCC: hepatocellular carcinoma

HOMA-IR: homeostatic mode assessment of insulin resistance

IL-6: interleukin-6

Ile: isoleucine

IncRNAs: includinig long non-coding RNAs

LSM: liver stiffness measurement

LysoPC: lysophosphatidylcholines

Mac2bp: Mac-2 binding protein

MAFLD: metabolic associated fatty liver disease

MetS: metabolic syndrome

miR: micro RNA

MRE: magnetic resonance elastography

MRI: magnetic resonance imaging

MRS: magnetic resonance spectroscopy

NAFL: non-alcoholic fatty liver

NAFLD: non-alcoholic fatty liver disease

NASH: non-alcoholic steatohepatitis

NFS: NAFLD fibrosis score

NPV: negative predictive value

OSAS: obstructive sleep apnea syndrome

PDFF: proton density fat fraction

PIIINP: N-termynal type III collagen propeptide

PLT: platelets

PNPLA3: patatin-like phospholipase domain containing 3 protein

PPV: positive predictive value

Pro-C3: type III collagen formation

pSWE: point shear wave elastography

ROI: region of interest

Se: sensitivity 
Ser: serine

SNP IFNL 4: single nucleotide polymorphism interferon lamba 4

Sp: specificity

T2DM: type 2 diabetes mellitus

TE: transient elastography

TG: triglycerides

TGFB2: transforming grow factor beta 2

TIMP-1: tissue inhibitor of matrix metalloproteinase 1

Tyr: tyrosine

US: ultrasound

VCTE: vibration controlled transient elastography

\section{Declarations}

\section{Acknowledgments}

The authors thank Giulio Mirabella for his contribution in the graphic support of the production of the figures in this paper.

\section{Author contributions}

VR, MM, AA and MP contributed conception and design of the study, VR and MM retrieved and selected the bibliographic references, VR and MM wrote the first draft of the manuscript, AA and MP revised it and wrote specific sessions. All authors contributed to manuscript revision, read and approved the submitted version.

\section{Conflicts of interest}

The authors declare that they have no conflicts of interest.

Ethical approval

Not applicable.

\section{Consent to participate}

Not applicable.

\section{Consent to publication}

Not applicable.

Availability of data and materials

Not applicable.

\section{Funding}

Not applicable.

\section{Copyright}

(C) The Author(s) 2020.

\section{References}

1. Masarone M, Federico A, Abenavoli L, Loguercio C, Persico M. Non alcoholic fatty liver: epidemiology and natural history. Rev Recent Clin Trials. 2014;9:126-33. 
2. Chalasani N, Younossi Z, Lavine JE, Charlton M, Cusi K, Rinella M, et al. The diagnosis and management of nonalcoholic fatty liver disease: practice guidance from the American Association for the Study of Liver Diseases. Hepatology. 2018;67:328-57.

3. Nascimbeni F, Pais R, Bellentani S, Day CP, Ratziu V, Loria P, et al. From NAFLD in clinical practice to answers from guidelines. J Hepatol. 2013;59:859-71.

4. Ertle J, Dechêne A, SowaJP, PenndorfV, Herzer K, Kaiser G, et al. Non-alcoholic fatty liver disease progresses to hepatocellular carcinoma in the absence of apparent cirrhosis. Int J Cancer. 2011;128:2436-43.

5. Younossi ZM, Koenig AB, Abdelatif D, Fazel Y, Henry L, Wymer M. Global epidemiology of nonalcoholic fatty liver disease-Meta-analytic assessment of prevalence, incidence, and outcomes. Hepatology. 2016;64:73-84.

6. Masarone M, Rosato V, Aglitti A, Bucci T, Caruso R, Salvatore T, et al. Liver biopsy in type 2 diabetes mellitus: steatohepatitis represents the sole feature of liver damage. PLoS One. 2017;12:e0178473.

7. Younossi ZM, Tampi R, Priyadarshini M, Nader F, Younossi IM, Racila A. Burden of illness and economic model for patients with nonalcoholic steatohepatitis in the United States. Hepatology. 2019;69:564-72.

8. Tran TT, Changsri C, Shackleton CR, Poordad FF, Nissen NN, Colquhoun S, et al. Living donor liver transplantation: histological abnormalities found on liver biopsies of apparently healthy potential donors. J Gastroenterol Hepatol. 2006;21:381-3.

9. Minervini MI, Ruppert K, Fontes P, Volpes R, Vizzini G, de Vera ME, et al. Liver biopsy findings from healthy potential living liver donors: reasons for disqualification, silent diseases and correlation with liver injury tests. J Hepatol. 2009;50:501-10.

10. Yilmaz Y, Kani HT, Demirtas CO, Kaya E, Sapmaz AF, Qutranji L, et al. Growing burden of nonalcoholic fatty liver disease in Turkey: a single-center experience. Turk J Gastroenterol. 2019;30:892-8.

11. Rosato V, Masarone M, Dallio M, Federico A, Aglitti A, Persico M. NAFLD and extra-hepatic comorbidities: current evidence on a multi-organ metabolic syndrome. Int J Environ Res Public Health. 2019;16:3415.

12. Singh S, Allen AM, Wang Z, Prokop LJ, Murad MH, Loomba R. Fibrosis progression in nonalcoholic fatty liver vs. nonalcoholic steatohepatitis: a systematic review and meta-analysis of paired-biopsy studies. Clin Gastroenterol Hepatol. Clin Gastroenterol Hepatol. 2015;13:643-54.e9.

13. Younossi Z, Tacke F, Arrese M, Chander Sharma B, Mostafa I, Bugianesi E, et al. Global perspectives on nonalcoholic fatty liver disease and nonalcoholic steatohepatitis. Hepatology. 2019;69:2672-82.

14. Noureddin M, Vipani A, Bresee C, Todo T, Kim IK, Alkhouri N, et al. NASH leading cause of liver transplant in women: updated analysis of indications for liver transplant and ethnic and gender variances. Am J Gastroenterol. 2018;113:1649-59.

15. Younossi Z, Stepanova M, Ong JP, Jacobson IM, Bugianesi E, Duseja A, et al. Nonalcoholic steatohepatitis is the fastest growing cause of hepatocellular carcinoma in liver transplant candidates. Clin Gastroenterol Hepatol. 2019;17:748-55.e3.

16. Kleiner DE, Brunt EM, Wilson LA, Behling C, Guy C, Contos M, et al. Association of histologic disease activity with progression of nonalcoholic fatty liver disease. JAMA Netw Open. 2019;2:e1912565.

17. Ratziu V, Charlotte F, Heurtier A, Gombert S, Giral P, Bruckert E, et al. Sampling variability of liver biopsy in nonalcoholic fatty liver disease. Gastroenterology. 2005;128:1898-906.

18. European Association for the Study of the Liver (EASL); European Association for the Study of Diabetes (EASD); European Association for the Study of Obesity (EASO). EASL-EASD-EASO Clinical Practice Guidelines for the management of non-alcoholic fatty liver disease. J Hepatol. 2016;64:1388-402.

19. Cook N, Geier A, Schmid A, Hirschfield G, Kautz A, Schattenberg JM, et al. Assessing physician preferences on future therapeutic options and diagnostic practices in non-alcoholic steatohepatitis. JHEP Rep. 2020;2:100081.

20. European Association for Study of Liver; Asociacion Latinoamericana para el Estudio del Higado. EASL-ALEH Clinical Practice Guidelines: non-invasive tests for evaluation of liver disease severity and prognosis. J Hepatol. 2015;63:237-64.

21. Angulo P. Long-term mortality in nonalcoholic fatty liver disease: is liver histology of any prognostic significance? Hepatology. 2010;51:373-5. 
22. Sheka AC, Adeyi O, Thompson J, Hameed B, Crawford PA, Ikramuddin S. Nonalcoholic steatohepatitis: a review. JAMA. 2020;323:1175-83.

23. Wright AP, Desai AP, Bajpai S, King LY, Sahani DV, Corey KE. Gaps in recognition and evaluation of incidentally identified hepatic steatosis. Dig Dis Sci. 2015;60:333-8.

24. Byrne CD, Targher G. NAFLD: a multisystem disease. J Hepatol. 2015;62:S47-64.

25. Ballestri S, Zona S, Targher G, Romagnoli D, Baldelli E, Nascimbeni F, et al. Nonalcoholic fatty liver disease is associated with an almost twofold increased risk of incident type 2 diabetes and metabolic syndrome. Evidence from a systematic review and meta-analysis. J Gastroenterol Hepatol. 2016;31:936-44.

26. Mantovani A, Byrne CD, Bonora E, Targher G. Nonalcoholic fatty liver disease and risk of incident type 2 diabetes: a meta-analysis. Diabetes Care. 2018;41:372-82.

27. Yilmaz Y. NAFLD in the absence of metabolic syndrome: different epidemiology, pathogenetic mechanisms, risk factors for disease progression? Semin Liver Dis. 2012;32:14-21.

28. Targher G, Byrne CD, Lonardo A, Zoppini G, Barbui C. Non-alcoholic fatty liver disease and risk of incident cardiovascular disease: a meta-analysis. J Hepatol. 2016;65:589-600.

29. Ballestri S, Nascimbeni F, Romagnoli D, Lonardo A. The independent predictors of non-alcoholic steatohepatitis and its individual histological features.: Insulin resistance, serum uric acid, metabolic syndrome, alanine aminotransferase and serum total cholesterol are a clue to pathogenesis and candidate targets for treatment. Hepatol Res. 2016;46:1074-87.

30. Ballestri S, Lonardo A, Bonapace S, Byrne CD, Loria P, Targher G. Risk of cardiovascular, cardiac and arrhythmic complications in patients with non-alcoholic fatty liver disease. World J Gastroenterol. 2014;20:1724-45.

31. Chen F, Esmaili S, Rogers GB, Bugianesi E, Petta S, Marchesini G, et al. Lean NAFLD: a distinct entity shaped by differential metabolic adaptation. Hepatology. 2020;71:1213-27.

32. Rastogi A, Shasthry SM, Agarwal A, Bihari C, Jain P, Jindal A, et al. Non-alcoholic fatty liver disease histological scoring systems: a large cohort single-center, evaluation study. APMIS. 2017;125:962-73.

33. Corey KE, Klebanoff MJ, Tramontano AC, Chung RT, Hur C. Screening for nonalcoholic steatohepatitis in individuals with type 2 diabetes: a cost-effectiveness analysis. Dig Dis Sci. 2016;61:2108-17.

34. Loomba R, Schork N, Chen CH, Bettencourt R, Bhatt A, Ang B, et al. Heritability of hepatic fibrosis and steatosis based on a prospective twin study. Gastroenterology. 2015;149:1784-93.

35. Eslam M, Newsome PN, Sarin SK, Anstee QM, Targher G, Romero-Gomez M, et al. A new definition for metabolic associated fatty liver disease: an international expert consensus statement. J Hepatol. 2020;73:202-9.

36. Mathiesen UL, Franzén LE, Frydén A, Foberg U, Bodemar G. The clinical significance of slightly to moderately increased liver transaminase values in asymptomatic patients. Scand J Gastroenterol. 1999;34:85-91.

37. Fracanzani AL, Valenti L, Bugianesi E, Andreoletti M, Colli A, Vanni E, et al. Risk of severe liver disease in nonalcoholic fatty liver disease with normal aminotransferase levels: a role for insulin resistance and diabetes. Hepatology. 2008;48:792-8.

38. Ulasoglu C, Enc FY, Kaya E, Yilmaz Y. Characterization of patients with biopsy-proven non-alcoholic fatty liver disease and normal aminotransferase levels. J Gastrointestin Liver Dis. 2019;28:427-31.

39. Verma S, Jensen D, Hart J, Mohanty SR. Predictive value of ALT levels for non-alcoholic steatohepatitis (NASH) and advanced fibrosis in non-alcoholic fatty liver disease (NAFLD). Liver Int. 2013;33:1398-405.

40. Pirvulescu I, Gheorghe L, Csiki I, Becheanu G, Dumbravă M, Fica S, et al. Noninvasive clinical model for the diagnosis of nonalcoholic steatohepatitis in overweight and morbidly obese patients undergoing bariatric surgery. Chirurgia (Bucur). 2012;107:772-9.

41. Sookoian S, Castaño G, Burgueño AL, Gianotti TF, Rosselli MS, Pirola CJ. A diagnostic model to differentiate simple steatosis from nonalcoholic steatohepatitis based on the likelihood ratio form of Bayes theorem. Clin Biochem. 2009;42:624-9.

42. Singh SP, Barik RK. NonInvasive biomarkers in nonalcoholic fatty liver disease: are we there yet? J Clin Exp Hepatol. 2020;10:88-98. 
43. Campos GM, Bambha K, Vittinghoff E, Rabl C, Posselt AM, Ciovica R, et al. A clinical scoring system for predicting nonalcoholic steatohepatitis in morbidly obese patients. Hepatology. 2008;47:1916-23.

44. Ulitsky A, Ananthakrishnan AN, Komorowski R, Wallace J, Surapaneni SN, Franco J, et al. A noninvasive clinical scoring model predicts risk of nonalcoholic steatohepatitis in morbidly obese patients. Obes Surg. 2010;20:685-91.

45. Dixon JB, Bhathal PS, O'Brien PE. Nonalcoholic fatty liver disease: predictors of nonalcoholic steatohepatitis and liver fibrosis in the severely obese. Gastroenterology. 2001;121:91-100.

46. Bril F, McPhaul MJ, Caulfield MP, Clark VC, Soldevilla-Pico C, Firpi-Morell RJ, et al. Performance of plasma biomarkers and diagnostic panels for nonalcoholic steatohepatitis and advanced fibrosis in patients with type 2 diabetes. Diabetes Care. 2020;43:290-7.

47. Ratziu V, Massard J, Charlotte F, Messous D, Imbert-Bismut F, Bonyhay L, et al. Diagnostic value of biochemical markers (FibroTest-FibroSURE) for the prediction of liver fibrosis in patients with nonalcoholic fatty liver disease. BMC Gastroenterol. 2006;6:6.

48. Poynard T, Ratziu V, Charlotte F, Messous D, Munteanu M, Imbert-Bismut F, et al. Diagnostic value of biochemical markers (NashTest) for the prediction of non alcoholo steato hepatitis in patients with nonalcoholic fatty liver disease. BMC Gastroenterol. 2006;6:34.

49. Poynard T, Lassailly G, Diaz E, Clement K, Caiazzo R, Tordjman J, et al. Performance of biomarkers FibroTest, ActiTest, SteatoTest, and NashTest in patients with severe obesity: meta analysis of individual patient data. PLoS One. 2012;7:e30325.

50. Ratziu V, Giral P, Munteanu M, Messous D, Mercadier A, Bernard M, et al. Screening for liver disease using non-invasive biomarkers (FibroTest, SteatoTest and NashTest) in patients with hyperlipidaemia. Aliment Pharmacol Ther. 2007;25:207-18.

51. Bril F, McPhaul MJ, Caulfield MP, Castille JM, Poynard T, Soldevila-Pico C, et al. Performance of the SteatoTest, ActiTest, NashTest and FibroTest in a multiethnic cohort of patients with type 2 diabetes mellitus. J Investig Med. 2019;67:303-11.

52. Wieckowska A, Papouchado BG, Li Z, Lopez R, Zein NN, Feldstein AE. Increased hepatic and circulating interleukin-6 levels in human nonalcoholic steatohepatitis. Am J Gastroenterol. 2008;103:1372-9.

53. Tarantino G, Conca P, Pasanisi F, Ariello M, Mastrolia M, Arena A, et al. Could inflammatory markers help diagnose nonalcoholic steatohepatitis? Eur J Gastroenterol Hepatol. 2009;21:504-11.

54. Grigorescu M, Crisan D, Radu C, Grigorescu MD, Sparchez Z, Serban A. A novel pathophysiologicalbased panel of biomarkers for the diagnosis of nonalcoholic steatohepatitis. J Physiol Pharmacol. 2012;63:347-53.

55. Machado MV, Coutinho J, Carepa F, Costa A, Proença H, Cortez-Pinto H. How adiponectin, leptin, and ghrelin orchestrate together and correlate with the severity of nonalcoholic fatty liver disease. Eur J Gastroenterol Hepatol. 2012;24:1166-72.

56. Kwok R, Tse YK, Wong GL, Ha Y, Lee AU, Ngu MC, et al. Systematic review with meta-analysis: noninvasive assessment of non-alcoholic fatty liver disease--the role of transient elastography and plasma cytokeratin-18 fragments. Aliment Pharmacol Ther. 2014;39:254-69.

57. Musso G, Gambino R, Cassader M, Pagano G. Meta-analysis: natural history of non-alcoholic fatty liver disease (NAFLD) and diagnostic accuracy of non-invasive tests for liver disease severity. Ann Med. 2011;43:617-49.

58. Feldstein AE, Wieckowska A, Lopez AR, Liu YC, Zein NN, McCullough AJ. Cytokeratin-18 fragment levels as noninvasive biomarkers for nonalcoholic steatohepatitis: a multicenter validation study. Hepatology. 2009;50:1072-8.

59. Younossi ZM, Page S, Rafiq N, Birerdinc A, Stepanova M, Hossain N, et al. A biomarker panel for nonalcoholic steatohepatitis (NASH) and NASH-related fibrosis. Obes Surg. 2011;21:431-9.

60. Anty R, Iannelli A, Patouraux S, Bonnafous S, Lavallard VJ, Senni-Buratti M, et al. A new composite model including metabolic syndrome, alanine aminotransferase and cytokeratin-18 for the diagnosis of nonalcoholic steatohepatitis in morbidly obese patients. Aliment Pharmacol Ther. 2010;32:1315-22. 
61. Cao W, Zhao C, Shen C, Wang Y. Cytokeratin 18, alanine aminotransferase, platelets and triglycerides predict the presence of nonalcoholic steatohepatitis. PLoS One. 2013;8:e82092.

62. Younossi ZM, Jarrar M, Nugent C, Randhawa M, Afendy M, Stepanova M, et al. A novel diagnostic biomarker panel for obesity-related nonalcoholic steatohepatitis (NASH). Obes Surg. 2008;18:1430-7.

63. Zheng KI, Liu WY, Pan XY, Ma HL, Zhu PW, Wu XX, et al. Combined and sequential non-invasive approach to diagnosing non-alcoholic steatohepatitis in patients with non-alcoholic fatty liver disease and persistently normal alanine aminotransferase levels. BMJ Open Diabetes Res Care. 2020;8:e001174.

64. Kamada Y, Ono M, Hyogo H, Fujii H, Sumida Y, Mori K, et al. A novel noninvasive diagnostic method for nonalcoholic steatohepatitis using two glycobiomarkers. Hepatology. 2015;62:1433-43.

65. Verhaegh P, Bavalia R, Winkens B, Masclee A, Jonkers D, Koek G. Noninvasive tests do not accurately differentiate nonalcoholic steatohepatitis from simple steatosis: a systematic review and meta-analysis. Clin Gastroenterol Hepatol. 2018;16:837-61.

66. Carter A, Mann JP. The clinical relevance of differentiating nonalcoholic steatohepatitis from simple steatosis. Clin Gastroenterol Hepatol. 2018;16:596.

67. Dulai PS, Singh S, Patel J, Soni M, Prokop LJ, Younossi Z, et al. Increased risk of mortality by fibrosis stage in nonalcoholic fatty liver disease: systematic review and meta-analysis. Hepatology. 2017;65:1557-65.

68. Taylor RS, Taylor RJ, Bayliss S, Hagström H, Nasr P, Schattenberg JM, et al. Association between fibrosis stage and outcomes of patients with nonalcoholic fatty liver disease: a systematic review and metaanalysis. Gastroenterology. 2020;158:1611-25.e12.

69. Jaruvongvanich $\mathrm{V}$, Wijarnpreecha K, Ungprasert P. The utility of NAFLD fibrosis score for prediction of mortality among patients with nonalcoholic fatty liver disease: a systematic review and meta-analysis of cohort study. Clin Res Hepatol Gastroenterol. 2017;41:629-34.

70. Simon TG, Corey KE, Cannon CP, Blazing M, Park JG, O'Donoghue ML, et al. The nonalcoholic fatty liver disease (NAFLD) fibrosis score, cardiovascular risk stratification and a strategy for secondary prevention with ezetimibe. Int J Cardiol. 2018;270:245-52.

71. Cai J, Zhang XJ, Ji YX, Zhang P, She ZG, Li H. Nonalcoholic fatty liver disease pandemic fuels the upsurge in cardiovascular diseases. Circ Res. 2020;126:679-704.

72. Ballestri S, Capitelli M, Fontana MC, Arioli D, Romagnoli E, Graziosi C, et al. Direct oral anticoagulants in patients with liver disease in the era of non-alcoholic fatty liver disease global epidemic: a narrative review. Adv Ther. 2020;37:1910-32.

73. Adler M, Gulbis B, Moreno C, Evrard S, Verset G, Golstein P, et al. The predictive value of FIB-4 versus FibroTest, APRI, FibroIndex and Forns index to noninvasively estimate fibrosis in hepatitis $\mathrm{C}$ and nonhepatitis C liver diseases. Hepatology. 2008;47:762-3; author reply 763.

74. Shah AG, Lydecker A, Murray K, Tetri BN, Contos MJ, Sanyal AJ, et al. Comparison of noninvasive markers of fibrosis in patients with nonalcoholic fatty liver disease. Clin Gastroenterol Hepatol. 2009;7:1104-12.

75. Angulo P, Hui JM, Marchesini G, Bugianesi E, George J, Farrell GC, et al. The NAFLD fibrosis score: a noninvasive system that identifies liver fibrosis in patients with NAFLD. Hepatology. 2007;45:846-54.

76. Harrison SA, Oliver D, Arnold HL, Gogia S, Neuschwander-Tetri BA. Development and validation of a simple NAFLD clinical scoring system for identifying patients without advanced disease. Gut. 2008;57:1441-7.

77. Xiao G, Zhu S, Xiao X, Yan L, Yang J, Wu G. Comparison of laboratory tests, ultrasound, or magnetic resonance elastography to detect fibrosis in patients with nonalcoholic fatty liver disease: a metaanalysis. Hepatology. 2017;66:1486-501.

78. Kaya E, Bakir A, Kani HT, Demirtas CO, Keklikkiran C, Yilmaz Y. Simple noninvasive scores are clinically useful to exclude, not predict, advanced fibrosis: a study in Turkish patients with biopsy-proven nonalcoholic fatty liver disease. Gut Liver. 2020;14:486-91.

79. Alkayyali T, Qutranji L, Kaya E, Bakir A, Yilmaz Y. Clinical utility of noninvasive scores in assessing advanced hepatic fibrosis in patients with type 2 diabetes mellitus: a study in biopsy-proven nonalcoholic fatty liver disease. Acta Diabetol. 2020;57:613-8.

80. Alexander M, Loomis AK, Fairburn-Beech J, van der Lei J, Duarte-Salles T, Prieto-Alhambra D, et al. Realworld data reveal a diagnostic gap in non-alcoholic fatty liver disease. BMC Med. 2018;16:130. 
81. McPherson S, Hardy T, Dufour JF, Petta S, Romero-Gomez M, Allison M, et al. Age as a confounding factor for the accurate non-invasive diagnosis of advanced NAFLD fibrosis. Am J Gastroenterol. 2017;112:740-51.

82. Doycheva I, Shaker M, Allende D, Lopez R, Watt KD, Alkhouri N. Low utility of noninvasive fibrosis scores in young adults with nonalcoholic fatty liver disease. Am J Gastroenterol. 2017;112:652-3.

83. Shah S, Dhami-Shah H, Kamble S, Shukla A. FIB-4 cut-off of 1.3 may be inappropriate in a primary care referral pathway for patients with non-alcoholic fatty liver disease. J Hepatol. 2020;73:216-7.

84. Ampuero J, Pais R, Aller R, Gallego-Durán R, Crespo J, García-Monzón C, et al. Development and validation of hepamet fibrosis scoring system-a simple, noninvasive test to identify patients with nonalcoholic fatty liver disease with advanced fibrosis. Clin Gastroenterol Hepatol. 2020;18:216-25.e5.

85. Cales P, Lainé F, Boursier J, Deugnier Y, Moal V, Oberti F, et al. Comparison of blood tests for liver fibrosis specific or not to NAFLD. J Hepatol. 2009;50:165-73.

86. Boursier J, Guillaume M, Leroy V, Irlès M, Roux M, Lannes A, et al. New sequential combinations of non-invasive fibrosis tests provide an accurate diagnosis of advanced fibrosis in NAFLD. J Hepatol. 2019;71:389-96.

87. Poynard T, Morra R, Halfon P, Castera L, Ratziu V, Imbert-Bismut F, et al. Meta-analyses of FibroTest diagnostic value in chronic liver disease. BMC Gastroenterol. 2007;7:40.

88. Munteanu M, Pais R, Peta V, Deckmyn O, Moussalli J, Ngo Y, et al. Long-term prognostic value of the FibroTest in patients with non-alcoholic fatty liver disease, compared to chronic hepatitis $\mathrm{C}, \mathrm{B}$, and alcoholic liver disease. Aliment Pharmacol Ther. 2018;48:1117-27.

89. Bush AM, Torres DM. Editorial: FibroTest to predict liver-related mortality in NAFLD. Should this change the diagnostic algorithm in NAFLD? Aliment Pharmacol Ther. 2018;48:1319-20.

90. Adams LA, George J, Bugianesi E, Rossi E, De Boer WB, van der Poorten D, et al. Complex non-invasive fibrosis models are more accurate than simple models in non-alcoholic fatty liver disease. J Gastroenterol Hepatol. 2011;26:1536-43.

91. Tanwar S, Trembling PM, Guha IN, Parkes J, Kaye P, Burt AD, et al. Validation of terminal peptide of procollagen III for the detection and assessment of nonalcoholic steatohepatitis in patients with nonalcoholic fatty liver disease. Hepatology. 2013;57:103-11.

92. Guha IN, Parkes J, Roderick P, Chattopadhyay D, Cross R, Harris S, et al. Noninvasive markers of fibrosis in nonalcoholic fatty liver disease: validating the European liver fibrosis panel and exploring simple markers. Hepatology. 2008;47:455-60.

93. Vali Y, Lee J, Boursier J, Spijker R, Löffler J, Verheij J, et al. Enhanced liver fibrosis test for the noninvasive diagnosis of fibrosis in patients with NAFLD: a systematic review and meta-analysis. J Hepatol. 2020;73:252-62.

94. Daniels SJ, Leeming DJ, Eslam M, Hashem AM, Nielsen MJ, Krag A, et al. ADAPT: an algorithm incorporating PRO-C3 accurately identifies patients with NAFLD and advanced fibrosis. Hepatology. 2019;69:1075-86.

95. Boyle M, Tiniakos D, Schattenberg JM, Ratziu V, Bugianessi E, Petta S, et al. Performance of the PRO-C3 collagen neo-epitope biomarker in non-alcoholic fatty liver disease. JHEP Rep. 2019;1:188-98.

96. Loomba R, Jain A, Diehl AM, Guy CD, Portenier D, Sudan R, et al. Validation of serum test for advanced liver fibrosis in patients with nonalcoholic steatohepatitis. Clin Gastroenterol Hepatol. 2019;17:1867-76.e3.

97. Aida Y, Abe H, Tomita Y, Nagano T, Seki N, Sugita T, et al. Serum immunoreactive collagen IV detected by monoclonal antibodies as a marker of severe fibrosis in patients with non-alcoholic fatty liver disease. J Gastrointestin Liver Dis. 2015;24:61-8.

98. Okanoue T, Ebise H, Kai T, Mizuno M, Shima T, Ichihara J, et al. A simple scoring system using type IV collagen 7S and aspartate aminotransferase for diagnosing nonalcoholic steatohepatitis and related fibrosis. J Gastroenterol. 2018;53:129-39.

99. Sumida Y, Yoneda M, Hyogo H, Yamaguchi K, Ono M, Fujii H, et al. A simple clinical scoring system using ferritin, fasting insulin, and type IV collagen 7S for predicting steatohepatitis in nonalcoholic fatty liver disease. J Gastroenterol. 2011;46:257-68. 
100. Ogawa Y, Honda Y, Kessoku T, Tomeno W, Imajo K, Yoneda M, et al. Wisteria floribunda agglutinin-positive Mac-2-binding protein and type 4 collagen 7S: useful markers for the diagnosis of significant fibrosis in patients with non-alcoholic fatty liver disease. J Gastroenterol Hepatol. 2018;33:1795-803.

101. Palmentieri B, de Sio I, La Mura V, Masarone M, Vecchione R, Bruno S, et al. The role of bright liver echo pattern on ultrasound B-mode examination in the diagnosis of liver steatosis. Dig Liver Dis. 2006;38:485-9.

102. Hernaez R, Lazo M, Bonekamp S, Kamel I, Brancati FL, Guallar E, et al. Diagnostic accuracy and reliability of ultrasonography for the detection of fatty liver: a meta-analysis. Hepatology. 2011;54:1082-90.

103. Paige JS, Bernstein GS, Heba E, Costa EAC, Fereirra M, Wolfson T, et al. A pilot comparative study of quantitative ultrasound, conventional ultrasound, and MRI for predicting histology-determined steatosis grade in adult nonalcoholic fatty liver disease. AJR Am J Roentgenol. 2017;208:W168-77.

104. de Moura Almeida A, Cotrim HP, Barbosa DB, de Athayde LG, Santos AS, Bitencourt AG, et al. Fatty liver disease in severe obese patients: diagnostic value of abdominal ultrasound. World J Gastroenterol. 2008;14:1415-8.

105. Ballestri S, Nascimbeni F, Baldelli E, Marrazzo A, Romagnoli D, Targher G, et al. Ultrasonographic fatty liver indicator detects mild steatosis and correlates with metabolic/histological parameters in various liver diseases. Metabolism. 2017;72:57-65.

106. Jun BG, Park WY, Park EJ, Jang JY, Jeong SW, Lee SH, et al. A prospective comparative assessment of the accuracy of the FibroScan in evaluating liver steatosis. PLoS One. 2017;12:e0182784.

107. Younossi ZM, Loomba R, Anstee QM, Rinella ME, Bugianesi E, Marchesini G, et al. Diagnostic modalities for nonalcoholic fatty liver disease, nonalcoholic steatohepatitis, and associated fibrosis. Hepatology. 2018;68:349-60.

108. Reeder SB, Cruite I, Hamilton G, Sirlin CB. Quantitative assessment of liver fat with magnetic resonance imaging and spectroscopy. J Magn Reson Imaging. 2011;34:729-49.

109. Middleton MS, Heba ER, Hooker CA, Bashir MR, Fowler KJ, Sandrasegaran K, et al. Agreement between magnetic resonance imaging proton density fat fraction measurements and pathologist-assigned steatosis grades of liver biopsies from adults with nonalcoholic steatohepatitis. Gastroenterology. 2017;153:753-61.

110. Noureddin M, Lam J, Peterson MR, Middleton M, Hamilton G, Le TA, et al. Utility of magnetic resonance imaging versus histology for quantifying changes in liver fat in nonalcoholic fatty liver disease trials. Hepatology. 2013;58:1930-40.

111. Loomba R, Neuschwander-Tetri BA, Sanyal A, Chalasani N, Diehl AM, Terrault N, et al. Multicenter validation of association between decline in MRI-PDFF and histologic response in nonalcoholic steatohepatitis. Hepatology. 2020; [Epub ahead of print].

112. Castera L, Friedrich-Rust M, Loomba R. Noninvasive assessment of liver disease in patients with nonalcoholic fatty liver disease. Gastroenterology. 2019;156:1264-81.e4.

113. Singh S, Venkatesh SK, Wang Z, Miller FH, Motosugi U, Low RN, et al. Diagnostic performance of magnetic resonance elastography in staging liver fibrosis: a systematic review and meta-analysis of individual participant data. Clin Gastroenterol Hepatol. 2015;13:440-51.e6.

114. Loomba R, Cui J, Wolfson T, Haufe W, Hooker J, Szeverenyi N, et al. Novel 3D magnetic resonance elastography for the noninvasive diagnosis of advanced fibrosis in NAFLD: a prospective study. Am J Gastroenterol. 2016;111:986-94.

115. Wagner M, Corcuera-Solano I, Lo G, Esses S, Liao J, Besa C, et al. Technical failure of MR elastography examinations of the liver: experience from a large single-center study. Radiology. 2017;284:401-12.

116. Chen J, Yin M, Talwalkar JA, Oudry J, Glaser KJ, Smyrk TC, et al. Diagnostic performance of MR elastography and vibration-controlled transient elastography in the detection of hepatic fibrosis in patients with severe to morbid obesity. Radiology. 2017;283:418-28.

117. Banerjee R, Pavlides M, Tunnicliffe EM, Piechnik SK, Sarania N, Philips R, et al. Multiparametric magnetic resonance for the non-invasive diagnosis of liver disease. J Hepatol. 2014;60:69-77. 
118. Pavlides M, Banerjee R, Tunnicliffe EM, Kelly C, Collier J, Wang LM, et al. Multiparametric magnetic resonance imaging for the assessment of non-alcoholic fatty liver disease severity. Liver Int. 2017;37:1065-73.

119. Yin M, Glaser KJ, Manduca A, Mounajjed T, Malhi H, Simonetto DA, et al. Distinguishing between hepatic inflammation and fibrosis with MR elastography. Radiology. 2017;284:694-705.

120. Sandrin L, Fourquet B, Hasquenoph JM, Yon S, Fournier C, Mal F, et al. Transient elastography: a new noninvasive method for assessment of hepatic fibrosis. Ultrasound Med Biol. 2003;29:1705-13.

121. Dietrich CF, Bamber J, Berzigotti A, Bota S, Cantisani V, Castera L, et al. EFSUMB Guidelines and Recommendations on the Clinical Use of Liver Ultrasound Elastography, Update 2017 (Long Version). Ultraschall Med. 2017;38:e16-47.

122. Petta S, Wong VW, Camma C, Hiriart JB, Wong GL, Vergniol J, et al. Serial combination of non-invasive tools improves the diagnostic accuracy of severe liver fibrosis in patients with NAFLD. Aliment Pharmacol Ther. 2017;46:617-27.

123. Boursier J, Vergniol J, Guillet A, Hiriart JB, Lannes A, Le Bail B, et al. Diagnostic accuracy and prognostic significance of blood fibrosis tests and liver stiffness measurement by FibroScan in non-alcoholic fatty liver disease. J Hepatol. 2016;65:570-8.

124. Inadomi C, Takahashi H, Ogawa Y, Oeda S, Imajo K, Kubotsu Y, et al. Accuracy of the enhanced liver fibrosis test, and combination of the enhanced liver fibrosis and non-invasive tests for the diagnosis of advanced liver fibrosis in patients with non-alcoholic fatty liver disease. Hepatol Res. 2020;50:682-92.

125. Newsome PN, Sasso M, Deeks JJ, Paredes A, Boursier J, Chan WK, et al. FibroScan-AST (FAST) score for the non-invasive identification of patients with non-alcoholic steatohepatitis with significant activity and fibrosis: a prospective derivation and global validation study. Lancet Gastroenterol Hepatol. 2020;5:362-73.

126. Hsu C, Caussy C, Imajo K, Chen J, Singh S, Kaulback K, et al. Magnetic resonance vs. transient elastography analysis of patients with nonalcoholic fatty liver disease: a systematic review and pooled analysis of individual participants. Clin Gastroenterol Hepatol. 2019;17:630-7.e8.

127. Sasso M, Beaugrand M, de Ledinghen V, Douvin C, Marcellin P, Poupon R, et al. Controlled attenuation parameter (CAP): a novel VCTE ${ }^{\mathrm{TM}}$ guided ultrasonic attenuation measurement for the evaluation of hepatic steatosis: preliminary study and validation in a cohort of patients with chronic liver disease from various causes. Ultrasound Med Biol. 2010;36:1825-35.

128. Myers RP, Pollett A, Kirsch R, Pomier-Layrargues G, Beaton M, Levstik M, et al. Controlled attenuation parameter (CAP): a noninvasive method for the detection of hepatic steatosis based on transient elastography. Liver Int. 2012;32:902-10.

129. de Lédinghen V, Wong GL, Vergniol J, Chan HL, Hiriart JB, Chan AW, et al. Controlled attenuation parameter for the diagnosis of steatosis in non-alcoholic fatty liver disease. J Gastroenterol Hepatol. 2016;31:848-55.

130. Karlas T, Petroff D, Sasso M, Fan JG, Mi YQ de Lédinghen V, et al. Individual patient data meta-analysis of controlled attenuation parameter (CAP) technology for assessing steatosis. J Hepatol. 2017;66:1022-30.

131. Chan WK, Nik Mustapha NR, Wong GL, Wong VW, Mahadeva S. Controlled attenuation parameter using the FibroScan(R) XL probe for quantification of hepatic steatosis for non-alcoholic fatty liver disease in an Asian population. United European Gastroenterol J. 2017;5:76-85.

132. de Lédinghen V, Hiriart JB, Vergniol J, Merrouche W, Bedossa P, Paradis V. Controlled attenuation parameter (CAP) with the XL probe of the Fibroscan $®$ : a comparative study with the M probe and liver biopsy. Dig Dis Sci. 2017;62:2569-77.

133. Park CC, Nguyen P, Hernandez C, Bettencourt R, Ramirez K, Fortney L, et al. Magnetic resonance elastography $v s$. transient elastography in detection of fibrosis and noninvasive measurement of steatosis in patients with biopsy-proven nonalcoholic fatty liver disease. Gastroenterology. 2017;152:598-607.e2.

134. Friedrich-Rust M, Poynard T, Castera L. Critical comparison of elastography methods to assess chronic liver disease. Nat Rev Gastroenterol Hepatol. 2016;13:402-11. 
135. Nierhoff J, Chávez Ortiz AA, Herrmann E, Zeuzem S, Friedrich-Rust M. The efficiency of acoustic radiation force impulse imaging for the staging of liver fibrosis: a meta-analysis. Eur Radiol. 2013;23:3040-53.

136. Liu H, Fu J, Hong R, Liu L, Li F. Acoustic radiation force impulse elastography for the non-invasive evaluation of hepatic fibrosis in non-alcoholic fatty liver disease patients: a systematic review \& metaanalysis. PLoS One. 2015;10:e0127782.

137. Cui J, Heba E, Hernandez C, Haufe W, Hooker J, Andre MP, et al. Magnetic resonance elastography is superior to acoustic radiation force impulse for the diagnosis of fibrosis in patients with biopsy-proven nonalcoholic fatty liver disease: a prospective study. Hepatology. 2016;63:453-61.

138. Herrmann E, de Lédinghen V, Cassinotto C, Chu WC, Leung VY, Ferraioli G, et al. Assessment of biopsyproven liver fibrosis by two-dimensional shear wave elastography: an individual patient data-based meta-analysis. Hepatology. 2018;67:260-72.

139. Cassinotto C, Boursier J, de Lédinghen V, Lebigot J, Lapuyade B, Cales P, et al. Liver stiffness in nonalcoholic fatty liver disease: a comparison of supersonic shear imaging, FibroScan, and ARFI with liver biopsy. Hepatology. 2016;63:1817-27.

140. Furlan A, Tublin ME, Yu L, Chopra KB, Lippello A, Behari J. Comparison of 2D shear wave elastography, transient elastography, and MR elastography for the diagnosis of fibrosis in patients with nonalcoholic fatty liver disease. AJR Am J Roentgenol. 2020;214:W20-6.

141. Gorden DL, Myers DS, Ivanova PT, Fahy E, Maurya MR, Gupta S, et al. Biomarkers of NAFLD progression: a lipidomics approach to an epidemic. J Lipid Res. 2015;56:722-36.

142. Loomba R, Quehenberger O, Armando A, Dennis EA. Polyunsaturated fatty acid metabolites as novel lipidomic biomarkers for noninvasive diagnosis of nonalcoholic steatohepatitis. J Lipid Res. 2015;56:185-92.

143. Zhou Y, Orešič M, Leivonen M, Gopalacharyulu P, Hyysalo J, Arola J, et al. Noninvasive detection of nonalcoholic steatohepatitis using clinical markers and circulating levels of lipids and metabolites. Clin Gastroenterol Hepatol. 2016;14:1463-72.e6.

144. Hyysalo J, Männistö VT, Zhou Y, Arola J, Kärjä V, Leivonen M, et al. A population-based study on the prevalence of NASH using scores validated against liver histology. J Hepatol. 2014;60:839-46.

145. Bell LN, Theodorakis JL, Vuppalanchi R, Saxena R, Bemis KG, Wang M, et al. Serum proteomics and biomarker discovery across the spectrum of nonalcoholic fatty liver disease. Hepatology. 2010;51:111-20.

146. Wood GC, Chu X, Argyropoulos G, Benotti P, Rolston D, Mirshahi T, et al. A multi-component classifier for nonalcoholic fatty liver disease (NAFLD) based on genomic, proteomic, and phenomic data domains. Sci Rep. 2017;7:43238.

147. Moolla A, de Boer J, Pavlov D, Amin A, Taylor A, Gilligan L, et al. Accurate non-invasive diagnosis and staging of non-alcoholic fatty liver disease using the urinary steroid metabolome. Aliment Pharmacol Ther. 2020;51:1188-97.

148. Qi S, Xu D, Li Q Xie N, Xia J, Huo Q, et al. Metabonomics screening of serum identifies pyroglutamate as a diagnostic biomarker for nonalcoholic steatohepatitis. Clin Chim Acta. 2017;473:89-95.

149. Alonso C, Fernández-Ramos D, Varela-Rey M, Martínez-Arranz I, Navasa N, Van Liempd SM, et al. Metabolomic identification of subtypes of nonalcoholic steatohepatitis. Gastroenterology. 2017;152:1449-61.e7.

150. Mayo R, Crespo J, Martínez-Arranz I, Banales JM, Arias M, Mincholé I, et al. Metabolomic-based noninvasive serum test to diagnose nonalcoholic steatohepatitis: results from discovery and validation cohorts. Hepatol Commun. 2018;2:807-20.

151. Ferslew BC, Xie G, Johnston CK, Su M, Stewart PW, Jia W, et al. Altered bile acid metabolome in patients with nonalcoholic steatohepatitis. Dig Dis Sci. 2015;60:3318-28.

152. Chen C, Schmilovitz-Weiss H, Liu XE, Pappo O, Halpern M, Sulkes J, et al. Serum protein N-glycans profiling for the discovery of potential biomarkers for nonalcoholic steatohepatitis. J Proteome Res. 2009;8:463-70. 
153. Blomme B, Francque S, Trépo E, Libbrecht L, Vanderschaeghe D, Verrijken A, et al. N-glycan based biomarker distinguishing non-alcoholic steatohepatitis from steatosis independently of fibrosis. Dig Liver Dis. 2012;44:315-22.

154. Blomme B, Fitzpatrick E, Quaglia A, De Bruyne R, Dhawan A, Van Vlierberghe H. Serum protein $\mathrm{N}$-glycosylation in paediatric non-alcoholic fatty liver disease. Pediatr Obes. 2012;7:165-73.

155. Romeo S, Kozlitina J, Xing C, Pertsemlidis A, Cox D, Pennacchio LA, et al. Genetic variation in PNPLA3 confers susceptibility to nonalcoholic fatty liver disease. Nat Genet. 2008;40:1461-5.

156. Kozlitina J, Smagris E, Stender S, Nordestgaard BG, Zhou HH, Tybjaerg-Hansen A, et al. Exome-wide association study identifies a TM6SF2 variant that confers susceptibility to nonalcoholic fatty liver disease. Nat Genet. 2014;46:352-6.

157. Mancina RM, Dongiovanni P, Petta S, Pingitore P, Meroni M, Rametta R, et al. The MBOAT7-TMC4 variant rs641738 increases risk of nonalcoholic fatty liver disease in individuals of European descent. Gastroenterology. 2016;150:1219-30.e6.

158. Eslam M, Hashem AM, Romero-Gomez M, Berg T, Dore GJ, Mangia A, et al. FibroGENE: a gene-based model for staging liver fibrosis. J Hepatol. 2016;64:390-8.

159. Loomba R, Seguritan V, Li W, Long T, Klitgord N, Bhatt A, et al. Gut microbiome-based metagenomic signature for non-invasive detection of advanced fibrosis in human nonalcoholic fatty liver disease. Cell Metab. 2017;25:1054-62.e5.

160. Dong TS, Katzka W, Lagishetty V, Luu K, Hauer M, Pisegna J, et al. A microbial signature identifies advanced fibrosis in patients with chronic liver disease mainly due to NAFLD. Sci Rep. 2020;10:2771.

161. Schwimmer JB, Johnson JS, Angeles JE, Behling C, Belt PH, Borecki I, et al. Microbiome signatures associated with steatohepatitis and moderate to severe fibrosis in children with nonalcoholic fatty liver disease. Gastroenterology. 2019;157:1109-22.

162. Kornek M, Lynch M, Mehta SH, Lai M, Exley M, Afdhal NH, et al. Circulating microparticles as diseasespecific biomarkers of severity of inflammation in patients with hepatitis C or nonalcoholic steatohepatitis. Gastroenterology. 2012;143:448-58.

163. Szabo G, Csak T. Role of microRNAs in NAFLD/NASH. Dig Dis Sci. 2016;61:1314-24.

164. Liu XL, Pan Q, Zhang RN, Shen F, Yan SY, Sun C, et al. Disease-specific miR-34a as diagnostic marker of non-alcoholic steatohepatitis in a Chinese population. World J Gastroenterol. 2016;22:9844-52.

165. Liu CH, Ampuero J, Gil-Gómez A, Montero-Vallejo R, Rojas A, Muñoz-Hernández R, et al. miRNAs in patients with non-alcoholic fatty liver disease: a systematic review and meta-analysis. J Hepatol. 2018;69:1335-48.

166. Di Mauro S, Scamporrino A, Petta S, Urbano F, Filippello A, Ragusa M, et al. Serum coding and non-coding RNAs as biomarkers of NAFLD and fibrosis severity. Liver Int. 2019;39:1742-54. 